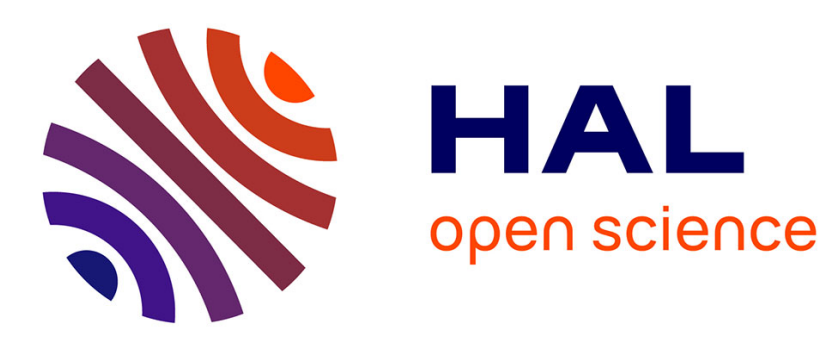

\title{
Adaptively Secure Non-interactive CCA-Secure Threshold Cryptosystems: Generic Framework and Constructions
}

\author{
Benoît Libert, Moti Yung
}

\section{To cite this version:}

Benoît Libert, Moti Yung. Adaptively Secure Non-interactive CCA-Secure Threshold Cryptosystems: Generic Framework and Constructions. Journal of Cryptology, 2020, 33, pp.1405-1441. 10.1007/s00145-020-09350-3 . hal-03116642

\section{HAL Id: hal-03116642 \\ https://hal.inria.fr/hal-03116642}

Submitted on 20 Jan 2021

HAL is a multi-disciplinary open access archive for the deposit and dissemination of scientific research documents, whether they are published or not. The documents may come from teaching and research institutions in France or abroad, or from public or private research centers.
L'archive ouverte pluridisciplinaire HAL, est destinée au dépôt et à la diffusion de documents scientifiques de niveau recherche, publiés ou non, émanant des établissements d'enseignement et de recherche français ou étrangers, des laboratoires publics ou privés. 


\title{
Adaptively Secure Non-Interactive CCA-Secure Threshold Cryptosystems: Generic Framework and Constructions ${ }^{\star}$
}

\author{
Benoît Libert ${ }^{1}$ and Moti Yung ${ }^{2} \star \star$ \\ ${ }^{1}$ CNRS, Laboratoire LIP (CNRS, ENSL, U. Lyon, Inria, UCBL), \\ ENS de Lyon (France) \\ ${ }^{2}$ Google and Columbia University (USA)
}

\begin{abstract}
In threshold cryptography, private keys are divided into $n$ shares, each one of which is given to a different server in order to avoid single points of failure. In the case of threshold public-key encryption, at least $t \leq n$ servers need to contribute to the decryption process. A threshold primitive is said robust if no coalition of $t$ malicious servers can prevent remaining honest servers from successfully completing private key operations. Non-interactive schemes, considered the most practical ones, allow servers to contribute to decryption without interactions. So far, most non-interactive threshold cryptosystems were only proved secure against static corruptions. In the adaptive corruption scenario (where the adversary can corrupt servers at any time, based on its complete view), all existing robust threshold encryption schemes that also resist chosen-ciphertext attacks (CCA) till recently require interaction in the decryption phase. A very specific method (in composite order groups) for getting rid of interaction was recently suggested, leaving the question of more generic frameworks and constructions with better security and, in particular, better flexibility (i.e., compatibility with distributed key generation).

This paper advances the state of the art and describes a general construction of adaptively secure robust non-interactive threshold cryptosystems with chosen-ciphertext security. We define the novel notion of all-but-one perfectly sound threshold hash proof systems that can be seen as (threshold) hash proof systems with publicly verifiable and simulation-sound proofs. We show that this notion generically implies threshold cryptosystems combining the aforementioned properties. Then, we provide efficient instantiations under well-studied assumptions in bilinear groups (e.g., in such groups of prime order). These instantiations have a tighter security proof in the single-challenge setting and are indeed compatible with distributed key generation protocols.
\end{abstract}

Keywords. Threshold cryptography, adaptive corruptions, public-key encryption, chosen-ciphertext security, non-interactivity, robustness.

\section{Introduction}

Threshold cryptography $[30,31,17]$ avoids single points of failure by splitting keys into $n>1$ shares which are held by servers in such a way that at least $t$ out of $n$ servers should contribute to private key operations. In $(t, n)$-threshold cryptosystems, an adversary breaking into up to $t-1$ servers should not jeopardize the security of the system.

Chosen-ciphertext security [64] (or IND-CCA for short) is widely recognized as the standard security notion for public-key encryption. Securely distributing the decryption procedure of CCAsecure public key schemes has proved to be a challenging task. As discussed in, e.g., [68,33], the difficulty is that decryption servers should return their partial decryption results, called "decryption shares", before knowing whether the incoming ciphertext is valid or not and partial decryptions of ill-formed ciphertexts may leak useful information to the adversary.

The first solution to this problem was put forth by Shoup and Gennaro [68] and it requires the

\footnotetext{
* This is the full version of a paper published in the proceedings of TCC 2012.

${ }^{\star \star}$ Part of this work was done while this author was in Snapchat.
} 
random oracle model [10], notably to render valid ciphertexts publicly recognizable. In the standard model, Canetti and Goldwasser [22] gave a threshold variant of the Cramer-Shoup encryption scheme [26]. Unfortunately, their scheme requires interaction among decryption servers to obtain robustness (i.e., ensure that no coalition of $t-1$ malicious servers can prevent uncorrupted servers from successfully decrypting) as well as to render invalid ciphertexts harmless. The approach of [22] consists in randomizing the decryption process in such a way that partial decryptions of invalid ciphertexts are uniformly random and thus meaningless to the adversary. To avoid the need to jointly generate randomizers at each decryption, shareholders can alternatively store a large number (i.e., proportional to the expected number of decryptions) of pre-shared secrets, which does not scale well. Cramer, Damgård and Ishai suggested [25] a method to generate randomizers without interaction but it is only efficient for a small number of servers.

Other threshold variants of Cramer-Shoup were suggested $[2,58]$ and Abe notably showed [2] how to achieve optimal resilience (namely, guarantee robustness as long as the adversary corrupts a minority of $t<n / 2$ servers) in the Canetti-Goldwasser system [22]. In the last decade or so, generic constructions of CCA-secure threshold cryptosystems with static security were put forth $[32,73]$.

Non-Interactive Schemes. As an application of the Canetti-Halevi-Katz (CHK) paradigm [23], Boneh, Boyen and Halevi [13] came up with the first fully non-interactive robust CCA-secure threshold cryptosystem with a security proof in the standard model: in their scheme, decryption servers can generate their decryption shares without any communication with other servers. Their scheme takes advantage of bilinear maps to publicly check the validity of ciphertexts, which considerably simplifies the task of proving security in the threshold setting. In addition, the validity of decryption shares can be verified in the same way, which provides robustness. Similar applications of the CHK methodology to threshold cryptography were studied in $[18,50]$.

Recently, Wee [73] defined a framework allowing to construct non-interactive threshold signatures and (chosen-ciphertext secure) threshold cryptosystems in a static corruption model. He left as an open problem the extension of his framework in the scenario of adaptive corruptions.

Adaptive Corruptions. Most threshold systems (including $[68,22,32,33,13]$ ) have been analyzed in a static corruption model, where the adversary chooses which servers it wants to corrupt before the scheme is set up. Unfortunately, adaptive adversaries - who can choose whom to corrupt at any time, as a function of their entire view of the protocol execution - are known (see, e.g., [24]) to be strictly stronger. As discussed in [22], properly dealing with adaptive corruptions often comes at some substantial expense like a lower resilience. For example, the Canetti-Goldwasser system can be proved robust and adaptively secure when the threshold $t$ is sufficiently small (typically, when $t=O\left(n^{1 / 2}\right)$ ), but, of course, supporting an optimal number of faulty servers is clearly preferable.

Assuming reliable erasures, Canetti et al. [21] devised adaptively secure protocols for the distributed generation of discrete-logarithm-based keys and DSA signatures. Their techniques were re-used later on [7] in proactive [62] RSA signatures. In 1999, Frankel, MacKenzie and Yung [34, 35] independently showed different methods to achieve adaptive security in the erasure-enabled setting.

The constructions of $[21,34,35]$ rely on a technique, called "single inconsistent player" (SIP), which inherently requires interaction. The SIP technique consists in transforming a $t$-out-of- $n$ secret sharing into an additive $t$-out-of- $t$ sharing of the same secret in such a way that, in the latter case, only one server has an internal state that cannot be consistently revealed to the adversary. As this player is randomly chosen upfront by the simulator among the $n$ severs, it only gets corrupted with probability bounded by $1 / 2$ and, should this undesirable event occur, the simulator can rewind 
the adversary back to one of its earlier states. After this rewinding operation, the simulator uses different random coins to simulate the adversary's view with the hope of avoiding a new corruption of the inconsistent player. Since the expected number of rewinds is only 2 , the reduction runs in polynomial time.

Jarecki and Lysyanskaya [44] subsequently extended the SIP technique to eliminate the need for erasures and provided an adaptively secure variant of the Canetti-Goldwasser threshold cryptosystem [22]. Their elegant scheme appeals to interactive zero-knowledge proofs but is designed to remain secure in concurrent environments. Unfortunately, their solution requires a fair amount of interaction among decryption servers. Abe and Fehr [5] showed how to dispense with zero-knowledge proofs in the Jarecki-Lysyanskaya construction so as to prove it secure in (a variant of) the universal composability framework but without completely eliminating interaction from the decryption procedure. As in most threshold variants of Cramer-Shoup, hedging against invalid decryption queries requires an interactive (though off-line) randomness generation phase for each ciphertext, unless many pre-shared secrets are stored.

In 2006, Almansa, Damgård and Nielsen [7] came up with a variant of Rabin's threshold RSA signatures [63] for which they proved adaptive security using the SIP technique and ideas from [34, 35]. Similar techniques were used in [69] to build adaptively secure threshold Waters signatures [70]. While the SIP technique does provide adaptively secure threshold protocols, it inherently requires interaction. The threshold schemes of $[34,35]$ proceed by turning a $(t, n)$ polynomial secret sharing into a $(t, t)$ additive secret sharing by first choosing a set of $t$ participants. If only one of these fails to provide a valid contribution to the private key operation, the entire protocol must restart from scratch. The threshold signatures of Almansa et al. [7] are slightly different in that, analogously to Rabin's technique [63], they proceed via an additive $(n, n)$-sharing of an RSA private key, the private RSA exponent $d$ being split into integers $d_{1}, \ldots, d_{n}$ summing to $d=\sum_{i=1}^{n} d_{i}$. In turn, each additive share $d_{i}$ is shared in a $(t, n)$ manner using a polynomial verifiable secret sharing, the $j$-th share $d_{i, j}$ of $d_{i}$ being given to server $j$. By doing so, if only one participant fails to provide a valid RSA signature share $H(M)^{d_{i}}$, the missing signature share can be re-constructed by running the reconstruction algorithm of the verifiable secret sharing scheme that was used to share $d_{i}$. As such, this approach is only non-interactive when all players are honest as a second round is needed to reconstruct missing multiplicative signature shares $H(M)^{d_{i}}$. Another consequence is that all servers have to store $\Theta(n)$ values, where $n$ is the total number of servers, as each player has to store a polynomial share of other players' additive share. Ideally, we would hope for solutions where each server only stores a constant number of elements, regardless of the number of involved servers.

The authors of this work [57] showed an adaptively secure variant of the Boneh-Boyen-Halevi construction [13] using groups of composite order and the dual system encryption approach [71,54] that was initially applied to identity-based encryption $[67,15]$. While it is a very encouraging and nice first step, the scheme of [57] is based on a very specific use of the Lewko-Waters techniques [54], which limits its applicability to composite order groups and makes it hard to combine with existing adaptively secure distributed key generation techniques. Also, the concrete security of this initial scheme is not optimal as its security reduction is related to the number of decryption queries made by the adversary. To solve these problems, we need a new approach and different methods to analyze the security of schemes.

Our COntribution. Motivated by an open question and needed extensions raised by Wee [73] and the work of [57], and in light of the long history of the problem outlined above, we define a general framework for constructing robust, adaptively secure and fully non-interactive threshold 
cryptosystems with chosen-ciphertext security. Our goal is to have simple and practical client/server protocols, as advocated in [68][Section 2.5], and even avoid the off-line interactive randomness generation stage which is usually needed in threshold versions of Cramer-Shoup [22].

While the Canetti-Halevi-Katz paradigm [23] is known $[13,18,50,4]$ to provide non-interactive threshold CCA2 security by leveraging the public verifiability of ciphertexts, it makes it hard to handle adaptive adversaries without introducing interaction. The main reason is that, in most instantiations, the public key information-theoretically commits servers to a unique private key share. As a result, the reduction is bound to a set of available private key shares which is usually determined at the start. To overcome this difficulty, we turn to hash proof systems (HPS) [27] and take advantage of the property that, in security reductions using the techniques of $[26,27]$, the simulator always knows the private keys, which is convenient to answering adaptive corruption queries (note that a similar observation was made by Dodis and Fazio [29] in a different context). In the threshold setting, when the reduction has to reveal the internal state of dynamically-corrupted servers, it is not bound to a particular set of available shares since it knows them all. However, one difficulty is that, unlike the constructions derived from the Canetti-Halevi-Katz paradigm $[13,18$, $50,4]$, valid ciphertexts are not recognizable from the public key alone. To solve this problem, we depart from [22] in that the validity of ciphertexts is made publicly verifiable - which eliminates the need to randomize the decryption operation - using non-interactive proofs satisfying a one-time simulation-soundness [65] property: in the reduction, the simulator should be able to generate a proof for a possibly false statement but the adversary should be statistically unable to do it on its own, even after having seen a fake proof.

To this end, we provide an efficient and natural method for upgrading Groth-Sahai proofs into one-time simulation-sound proof for linear multi-exponentiation equations. In a nutshell, the idea is to embed the verification key of a one-time signature into the Groth-Sahai CRS which is used to prove the statement. This is done in such a way that the Groth-Sahai CRS is perfectly sound for all one-time verification keys, except a specific one which allows simulating fake proofs using a trapdoor. In comparison with original Groth-Sahai proofs, our non-interactive proofs are only longer by the length of a one-time signature and its verification key. In order to instantiate our technique in a generic manner under different assumptions, we present an abstraction of our proof systems. Namely, we define the notion of all-but-one perfectly sound threshold hash proof systems that can be seen as (threshold) hash proof systems [27] with publicly verifiable proofs (as opposed to designed-verifier proofs used in traditional HPS [27]). More precisely, each proof is associated with a tag, in the same way as ciphertexts are associated with tags in $[59,50]$. Real public parameters are indistinguishable from alternative parameters that are generated in an all-but-one mode, which is only used in the security analysis. In the latter mode, non-interactive proofs are perfectly sound on all tags, except for a single specific tag where some trapdoor makes it possible to simulate proofs for false statements. While our primitive bears similarities with Wee's extractable hash proof systems $[72,73]$ (where hash proof systems are also associated with tags), it is different in that no extractability property is required and proofs are always used as proofs of membership rather than proofs of knowledge.

Using all-but-one perfectly sound threshold hash proof systems, we generically construct adaptively secure robust non-interactive threshold cryptosystems with optimal resilience. An additional benefit of this approach is to provide a better concrete security since the security proof requires a constant number of game transitions, whereas in [57], the number of games is proportional to the number of decryption queries. 
Then, we show three concrete instantiations of our framework using number theoretic assumptions in bilinear groups. The first one uses groups whose order is a product of two primes (whereas three primes are needed in [57]). Our second and third schemes rely on the Groth-Sahai proof systems [40] in their instantiations based on the Decision Linear [14] and symmetric eXternal Diffie-Hellman assumptions [66]. The latter two constructions operate over bilinear groups of prime order, which allows for a significantly better efficiency than composite order groups (as discussed in [36]) and makes them much easier to combine with known adaptively secure discrete-log-based distributed key generation protocols. For example, in the erasure-free setting, the protocols of [44, 5] can be used so as to eliminate the need for a trusted dealer at the same time as the reliance on reliable erasures. Indeed, in our constructions, only the dealer should be able to erase its secrets after the dealing phase.

Related and Subsequent Work. A first method for achieving simulation-sound Groth-Sahai proofs was proposed by Groth in [38]. Efficiency improvements were suggested by Chandran et al. [19] while Katz and Vaikuntanathan [49] obtained significantly shorter proofs in the case of one-time simulation-soundness. As will be discussed later on, our all-but-one proof systems yield shorter proofs than [49] by embedding a one-time signature verification key in the Groth-Sahai CRS. Concurrently to this work, Jutla and Roy [45] introduced a different method of achieving a (slightly relaxed but sufficient) notion of one-time simulation-soundness in Groth-Sahai proofs for linear multi-exponentiation equations. Instead of using one-time signatures, they combine hash proof systems [27] with publicly verifiable Groth-Sahai proofs for multi-exponentiation equations: in short, they rely on the idea that, for any adversarially generated proof, the private verification algorithm of the hash proof system should almost always agree with the public verification algorithm of Groth-Sahai proofs unless some assumption is broken. The Jutla-Roy technique turns out to be slightly more efficient than ours since they do not use one-time signatures.

Later on, Jutla and Roy [46] achieved significantly shorter proofs for linear equations in the quasi-adaptive setting, where the common reference string may depend on the language of which membership must be proved. This setting was even reported $[55,47,1,52]$ to enable constant-size proofs for the specific task of proving that a vector of group elements belongs to a linear subspace. In particular, the results of $[55,52]$ significantly improve upon the present paper in that they imply non-interactive threshold variants of Cramer-Shoup [26] where public verifiability is obtained by introducing only one more group element in ciphertexts.

Despite the advantages in efficiency of the quasi-adaptive setting, we believe our all-but-one proof systems to remain interesting today due to their properties and applicability: First, they ensure statistical soundness on all tags, except a specific one where they enable simulation. In contrast, the quasi-adaptive solutions of $[55,52]$ are restricted to provide computational soundness at any time. Secondly, our proof systems are not restricted to linear multi-exponentiation equations: for example, they still work for quadratic equations involving scalars. Moreover, they were found useful in the structure-preserving setting $[6,20]$, where ciphertexts can possibly serve as witnesses in Groth-Sahai proofs, so that CCA2 security should be achieved without any hash function evaluation. By extending the techniques of this work, Libert et al. [56] came up with a structure-preserving chosen-ciphertext-secure encryption scheme which readily enables threshold decryption. In terms of ciphertext length, their scheme also significantly improves upon the best previously known [3] structure-preserving CCA2-secure cryptosystem with publicly verifiable ciphertexts.

At a high level, the scheme of [56] exploits the fact that the all-but-one property of our proof system allows removing the need for a 2-universal hash proof system [27]. Since 2-universality is 
usually obtained using a hash function (which is incompatible with Groth-Sahai proofs), its elimination is one of the reasons why our framework can enable structure-preserving instantiations by introducing additional ideas. The schemes described in this paper are not structure-preserving since they interpret one-time signature verification keys as exponents. This difficulty was resolved in [56], where a special kind of structure-preserving commitment is used to support one-time verification keys made of group elements.

\section{Background and Definitions}

\subsection{Definitions for Threshold Public Key Encryption}

A non-interactive $(t, n)$-threshold encryption scheme is a set of algorithms with these specifications.

$\operatorname{Setup}(\lambda, t, n)$ : given a security parameter $\lambda$ and integers $t, n \in \operatorname{poly}(\lambda)$ (with $1 \leq t \leq n$ ) denoting the number of decryption servers $n$ and the decryption threshold $t$, this algorithm outputs $(P K, \mathbf{V K}, \mathbf{S K})$, where $P K$ is the public key, $\mathbf{S K}=\left(S K_{1}, \ldots, S K_{n}\right)$ is a vector of private-key shares and $\mathbf{V K}=\left(V K_{1}, \ldots, V K_{n}\right)$ is a vector of verification keys. Decryption server $i$ is given the private key share $\left(i, S K_{i}\right)$. For each $i \in\{1, \ldots, n\}$, the verification key $V K_{i}$ will be used to check the validity of decryption shares generated using $S K_{i}$.

$\operatorname{Encrypt}(P K, M)$ : is a randomized algorithm that, given a public key $P K$ and a plaintext $M$, outputs a ciphertext $C$.

Ciphertext-Verify $(P K, C)$ : takes as input a public key $P K$ and a ciphertext $C$. It outputs 1 if $C$ is deemed valid w.r.t. $P K$ and 0 otherwise.

Share-Decrypt $\left(P K, i, S K_{i}, C\right)$ : on input of a public key $P K$, a ciphertext $C$ and a privatekey share $\left(i, S K_{i}\right)$, this (possibly randomized) algorithm outputs a special symbol $(i, \perp)$ if Ciphertext-Verify $(P K, C)=0$. Otherwise, it outputs a decryption share $\mu_{i}=\left(i, \hat{\mu}_{i}\right)$.

Share-Verify $\left(P K, V K_{i}, C, \mu_{i}\right)$ : takes in $P K$, the verification key $V K_{i}$, a ciphertext $C$ and a purported decryption share $\mu_{i}=\left(i, \hat{\mu}_{i}\right)$. It outputs either 1 or 0 . In the former case, $\mu_{i}$ is said to be a valid decryption share. We adopt the convention that $(i, \perp)$ is an invalid decryption share.

Combine $\left(P K, \mathbf{V K}, C,\left\{\mu_{i}\right\}_{i \in S}\right)$ : given $P K, \mathbf{V K}, C$ and a subset $S \subset\{1, \ldots, n\}$ of size $t=|S|$ with decryption shares $\left\{\mu_{i}\right\}_{i \in S}$, this algorithm outputs either a plaintext $M$ or $\perp$ if the set contains invalid decryption shares.

Chosen-CIPHERTEXT SECURITy. We use a game-based definition of chosen-ciphertext security which is akin to the one of $[68,13]$ with the difference that the adversary can adaptively decide which parties it wants to corrupt.

Definition 1. A non-interactive $(t, n)$-Threshold Public Key Encryption scheme is secure against chosen-ciphertext attacks (or IND-CCA2 secure) and adaptive corruptions if no PPT adversary has non-negligible advantage in this game:

1. The challenger runs $\operatorname{Setup}(\lambda, t, n)$ to obtain a public key $P K$, a vector of private key shares $\mathbf{S K}=\left(S K_{1}, \ldots, S K_{n}\right)$ and verification keys $\mathbf{V K}=\left(V K_{1}, \ldots, V K_{n}\right)$. It gives $P K$ and $\mathbf{V K}$ to the adversary $\mathcal{A}$ and keeps $\mathbf{S K}$ to itself.

2 The adversary $\mathcal{A}$ adaptively makes the following kinds of queries:

- Corruption query: $\mathcal{A}$ chooses $i \in\{1, \ldots, n\}$ and obtains $S K_{i}$. No more than $t-1$ private key shares can be obtained by $\mathcal{A}$ in the whole game. 
- Decryption query: $\mathcal{A}$ chooses an index $i \in\{1, \ldots, n\}$ and a ciphertext $C$. The challenger replies with $\mu_{i}=\operatorname{Share-Decrypt}\left(P K, i, S K_{i}, C\right)$.

3. The adversary $\mathcal{A}$ chooses two equal-length messages $M_{0}, M_{1}$ and obtains $C^{\star}=\operatorname{Encrypt}\left(P K, M_{\beta}\right)$ for some random bit $\beta \stackrel{R}{\leftarrow}\{0,1\}$.

4. $\mathcal{A}$ makes further queries as in step 2 but is not allowed to make decryption queries on $C^{\star}$.

5. $\mathcal{A}$ outputs a bit $\beta^{\prime}$ and is deemed successful if $\beta^{\prime}=\beta$. As usual, $\mathcal{A}$ 's advantage is measured as the distance $\operatorname{Adv}(\mathcal{A})=\left|\operatorname{Pr}\left[\beta^{\prime}=\beta\right]-\frac{1}{2}\right|$.

Consistency. A $(t, n)$-Threshold Encryption scheme provides decryption consistency if no PPT adversary has non-negligible advantage in a three-stage game where stages 1 and 2 are identical to those of Definition 1 with the difference that the adversary $\mathcal{A}$ is allowed to obtain all private key shares (alternatively, $\mathcal{A}$ can directly obtain SK at the beginning of the game). In stage $3, \mathcal{A}$ outputs a ciphertext $C$ and two $t$-sets of decryption shares $\Gamma=\left\{\mu_{1}, \ldots, \mu_{t}\right\}$ and $\Gamma^{\prime}=\left\{\mu_{1}^{\prime}, \ldots, \mu_{t}^{\prime}\right\}$. The adversary $\mathcal{A}$ is declared successful if

1. Ciphertext-Verify $(P K, C)=1$.

2. $\Gamma$ and $\Gamma^{\prime}$ only consist of valid decryption shares.

3. Combine $(P K, \mathbf{V K}, C, \Gamma) \neq \mathbf{C o m b i n e}\left(P K, \mathbf{V K}, C, \Gamma^{\prime}\right)$.

We note that condition 1 prevents an adversary from trivially winning by outputting an invalid ciphertext, for which distinct sets of key shares may give different results. This definition of consistency is identical to the one of $[68,13]$ with the difference that $\mathcal{A}$ can adaptively corrupt servers.

\subsection{Hardness Assumptions in Composite Order Groups}

In one case, we appeal to groups $\left(\mathbb{G}, \mathbb{G}_{T}\right)$ of order $N=p_{1} p_{2}$, where $p_{1}$ and $p_{2}$ are primes, with a bilinear map $e: \mathbb{G} \times \mathbb{G} \rightarrow \mathbb{G}_{T}$ (i.e., for which $e\left(g^{a}, h^{b}\right)=e(g, h)^{a b}$ for any $g, h \in \mathbb{G}$ and $a, b \in \mathbb{Z}_{N}$ ). In the notations hereafter, for each $i \in\{1,2\}, \mathbb{G}_{p_{i}}$ stands for the subgroup of order $p_{i}$ in $\mathbb{G}$.

Definition 2 ([16]). In a group $\mathbb{G}$ of composite order $N$, the Subgroup Decision (SD) problem is given $\left(g \in \mathbb{G}_{p_{1}}, h \in \mathbb{G}\right)$ and $\eta$, to decide whether $\eta \in_{R} \mathbb{G}_{p_{1}}$ or $\eta \in_{R} \mathbb{G}$. The Subgroup Decision assumption states that, for any PPT distinguisher $\mathcal{D}$, the $S D$ problem is infeasible.

\subsection{Assumptions in Prime Order Groups}

We also use bilinear maps $e: \mathbb{G} \times \hat{\mathbb{G}} \rightarrow \mathbb{G}_{T}$ over groups of prime order $p$. We will work in symmetric pairing configurations, where $\mathbb{G}=\hat{\mathbb{G}}$, and sometimes in asymmetric configurations, where $\mathbb{G} \neq \hat{\mathbb{G}}$.

In the symmetric setting $\left(\mathbb{G}, \mathbb{G}_{T}\right)$, we rely on the following assumption.

Definition 3 ([14]). In a group $\mathbb{G}$ of prime order $p$, the Decision Linear Problem (DLIN) is to distinguish the distributions $\left(g, g^{a}, g^{b}, g^{a c}, g^{b d}, g^{c+d}\right)$ and $\left(g, g^{a}, g^{b}, g^{a c}, g^{b d}, g^{z}\right)$, with $a, b, c, d, z \stackrel{R}{\leftarrow} \mathbb{Z}_{p}$. The Decision Linear Assumption is the intractability of DLIN for any PPT distinguisher $\mathcal{D}$.

The problem amounts to deciding if vectors $\overrightarrow{g_{1}}=\left(g^{a}, 1, g\right), \overrightarrow{g_{2}}=\left(1, g^{b}, g\right)$ and $\overrightarrow{g_{3}}=\left(g^{a c}, g^{b d}, g^{\delta}\right)$ are linearly dependent (i.e., if $\delta=c+d$ ) or not.

In asymmetric bilinear groups $\left(\mathbb{G}, \hat{\mathbb{G}}, \mathbb{G}_{T}\right)$, we assume the hardness of the Decision Diffie-Hellman $(\mathrm{DDH})$ problem in $\mathbb{G}$ and $\hat{\mathbb{G}}$. This implies the unavailability of efficiently computable isomorphisms between $\hat{\mathbb{G}}$ and $\mathbb{G}$. This assumption is called Symmetric eXternal Diffie-Hellman (SXDH) assumption. Given vectors $\vec{u}_{1}=(g, h), \vec{u}_{2}=\left(g^{a}, h^{c}\right)$ in $\mathbb{G}^{2}$ or $\hat{\mathbb{G}}^{2}$, the SXDH assumption asserts the infeasibility of deciding whether $\vec{u}_{1}$ and $\vec{u}_{2}$ are linearly dependent (i.e., whether $a=c \bmod p$ ). 


\subsection{Groth-Sahai Proof Systems}

In the following notations, for equal-dimension vectors $\vec{A}$ and $\vec{B}$ containing group elements, $\vec{A} \cdot \vec{B}$ stands for their component-wise product.

In their instantiation based on the DLIN assumption in symmetric bilinear groups $\left(\mathbb{G}, \mathbb{G}_{T}\right)$, Groth-Sahai (GS) proofs [40] use a common reference string comprised of vectors $\overrightarrow{g_{1}}, \overrightarrow{g_{2}}, \overrightarrow{g_{3}} \in \mathbb{G}^{3}$, where $\overrightarrow{g_{1}}=\left(g_{1}, 1, g\right), \overrightarrow{g_{2}}=\left(1, g_{2}, g\right)$ for some $g_{1}, g_{2} \in \mathbb{G}$. A commitment to $X \in \mathbb{G}$ is obtained as $\vec{C}=(1,1, X) \cdot{\overrightarrow{g_{1}}}^{r} \cdot{\overrightarrow{g_{2}}}^{s} \cdot{\overrightarrow{g_{3}}}^{t}$ with $r, s, t \stackrel{R}{\leftarrow} \mathbb{Z}_{p}^{*}$. When proofs should be perfectly sound, ${\overrightarrow{g_{3}}}$ is set as $\overrightarrow{g_{3}}=\overrightarrow{g_{1}} \xi_{1} \cdot \overrightarrow{g_{2}} \xi_{2}$, with $\xi_{1}, \xi_{2} \stackrel{R}{\leftarrow} \mathbb{Z}_{p}^{*}$, so that $\vec{C}=\left(g_{1}^{r+\xi_{1} t}, g_{2}^{s+\xi_{2} t}, X \cdot g^{r+s+t\left(\xi_{1}+\xi_{2}\right)}\right)$ is a Boneh-BoyenShacham (BBS) encryption [14] that can be decrypted using $\alpha_{1}=\log _{g}\left(g_{1}\right), \alpha_{2}=\log _{g}\left(g_{2}\right)$. In the witness indistinguishability (WI) setting, $\overrightarrow{g_{1}}, \overrightarrow{g_{2}}, \overrightarrow{g_{3}}$ are linearly independent and $\vec{C}$ is a perfectly hiding commitment. Under the DLIN assumption, the two settings are indistinguishable.

To commit to an exponent $x \in \mathbb{Z}_{p}$, one computes $\vec{C}=\vec{\varphi}^{x} \cdot{\overrightarrow{g_{1}}}^{r} \cdot{\overrightarrow{g_{2}}}^{s}$, with $r, s \stackrel{R}{\leftarrow} \mathbb{Z}_{p}^{*}$, using a CRS comprising vectors $\vec{\varphi}, \overrightarrow{g_{1}}, \overrightarrow{g_{2}}$. In the soundness setting $\vec{\varphi}, \overrightarrow{g_{1}}, \overrightarrow{g_{2}}$ are linearly independent vectors (typically, one chooses $\vec{\varphi}=\overrightarrow{g_{3}} \cdot(1,1, g)$ where $\overrightarrow{g_{3}}={\overrightarrow{g_{1}}}^{\xi_{1}} \cdot{\overrightarrow{g_{2}}}^{\xi_{2}}$ ) whereas, in the WI setting, choosing $\vec{\varphi}=\overrightarrow{g_{1}} \vec{\xi}_{1} \cdot \vec{g}_{2} \xi_{2}$ gives a perfectly hiding commitment since $\vec{C}$ is always a BBS encryption of $1_{\mathbb{G}}$. On a perfectly sound CRS (where $\overrightarrow{g_{3}}=\overrightarrow{g_{1}} \vec{\xi}_{1} \cdot{\overrightarrow{g_{2}}}^{\xi_{2}}$ and $\vec{\varphi}=\overrightarrow{g_{3}} \cdot(1,1, g)$ ), commitments to exponents are not fully extractable since the trapdoor $\left(\alpha_{1}, \alpha_{2}\right)$ only allows recovering $g^{x}$ from $\vec{C}=\vec{\varphi}^{x} \cdot{\overrightarrow{g_{1}}}^{r} \cdot{\overrightarrow{g_{2}}}^{s}$.

To prove that committed variables satisfy certain relations, the techniques of [40] require one commitment per variable and one proof element per relation. Such efficient proofs notably exist for multi-exponentiation equations which are equations of the form

$$
\prod_{i=1}^{m} \mathcal{A}_{i}^{y_{i}} \cdot \prod_{j=1}^{n} \mathcal{X}_{j}^{b_{j}} \cdot \prod_{i=1}^{m} \cdot \prod_{j=1}^{n} \mathcal{X}_{j}^{y_{i} \gamma_{i j}}=T
$$

for variables $\mathcal{X}_{1}, \ldots, \mathcal{X}_{n} \in \mathbb{G}, y_{1}, \ldots, y_{m} \in \mathbb{Z}_{p}$ and constants $T, \mathcal{A}_{1}, \ldots, \mathcal{A}_{m} \in \mathbb{G}, b_{1}, \ldots, b_{n} \in \mathbb{Z}_{p}$ and $\gamma_{i j} \in \mathbb{G}$, for $i \in\{1, \ldots, m\}, j \in\{1, \ldots, n\}$.

Multi-exponentiation equations admit zero-knowledge proofs at no additional cost. On a simulated CRS (prepared for the WI setting), the trapdoor $\left(\xi_{1}, \xi_{2}\right)$ makes it possible to simulate proofs without knowing witnesses, and simulated proofs are perfectly indistinguishable from real proofs.

For linear equations (i.e., when $\gamma_{i j}=0$ for all $i, j$ ) depends on the form of the considered equation. Namely, linear multi-exponentiation equations of the type $\prod_{j=1}^{n} \mathcal{X}_{j}^{b_{j}}=T\left(\operatorname{resp} . \prod_{i=1}^{m} \mathcal{A}_{i}^{y_{i}}=T\right)$ demand 3 (resp. 2) group elements.

The Groth-Sahai techniques can also be instantiated in groups $\left(\mathbb{G}, \hat{\mathbb{G}}, \mathbb{G}_{T}\right)$ with an asymmetric bilinear map $e: \mathbb{G} \times \hat{\mathbb{G}} \rightarrow \mathbb{G}_{T}$, where $\mathbb{G} \neq \hat{\mathbb{G}}$. In this case, they rely on the Symmetric eXternal Diffie-Hellman assumption according to which the DDH problem is hard in both $\mathbb{G}$ and $\hat{\mathbb{G}}$. In this setting, we only use them to prove multi-exponentiation equations of the form $\prod_{i=1}^{m} \mathcal{A}_{i}^{y_{i}}=T$, for constants $\mathcal{A}_{1}, \ldots, \mathcal{A}_{m}, T \in \mathbb{G}$ and variables $y_{1}, \ldots, y_{m} \in \mathbb{Z}_{p}$. To this end, commitments to exponents $x \in \mathbb{Z}_{p}$ have to be computed in $\hat{\mathbb{G}}$. The common reference string includes vector $\vec{u}, \vec{u}_{1} \in \hat{\mathbb{G}}^{2}$ and commitments are calculated as per $\vec{C}=\vec{u}^{x} \cdot \vec{u}_{1}^{r}$, with $r \stackrel{R}{\leftarrow} \mathbb{Z}_{p}$. It is easy to see that the commitment $\vec{C}$ is perfectly binding if $\left(\vec{u}, \overrightarrow{u_{1}}\right)$ are linearly independent and perfectly hiding if $\vec{u} \in \operatorname{span}\left(\vec{u}_{1}\right)$. The corresponding proof for the equation $\prod_{i=1}^{m} \mathcal{A}_{i}^{y_{i}}=T$ will be perfectly sound if $\vec{u} \notin \operatorname{span}\left(\vec{u}_{1}\right)$ and perfectly WI if $\vec{u} \in \operatorname{span}\left(\vec{u}_{1}\right)$. In either case, the proof consists of a single element of $\mathbb{G}$. 


\section{All-But-One Perfectly Sound Threshold Hash Proof Systems}

Let $\mathcal{C}, \mathcal{K}$ and $\mathcal{K}^{\prime}$ be sets and let $\mathcal{V} \subset \mathcal{C}$ be a subset. Let also $\mathcal{R}$ be a space where random coins can be chosen. We mandate that $\mathcal{V}, \mathcal{K}, \mathcal{K}^{\prime}$ and $\mathcal{R}$ be of exponential size in $\lambda$, where $\lambda \in \mathbb{N}$ is a security parameter. In addition, $\mathcal{C}, \mathcal{V}$ and $\mathcal{C} \backslash \mathcal{V}$ should be efficiently samplable and we also require the set $\mathcal{K}$ to form a group for some binary operation, which is denoted by $\odot$ hereafter.

Intuitively, our encryption schemes will involve ciphertexts where certain components $\Phi$ live in $\mathcal{C}$. In all legitimate outputs of the encryption algorithm, we will have $\Phi \in \mathcal{V}$ while the security proof will involve elements $\Phi \in \mathcal{C} \backslash \mathcal{V}$. In the encryption algorithm, messages will consist of elements of the group $(\mathcal{K}, \odot)$, which will be hidden by a random one-time key $K \in \mathcal{K}$ obtained by running a public evaluation algorithm over $\Phi \in \mathcal{V}$. Upon decryption, the one-time key $K$ can be reconstructed from $t$ private evaluations $K_{i} \in \mathcal{K}^{\prime}$ of $\Phi \in \mathcal{V}$ using distinct private key shares $\mathrm{sk}_{i}$. At the same time, it should be information-theoretically infeasible to come up with $t$ distinct private evaluations of any $\Phi \in \mathcal{C} \backslash \mathcal{V}$, even given an arbitrary subset of $t-1$ private key shares.

An all-but-one perfectly sound threshold hash proof system for the $\operatorname{sets}\left(\mathcal{C}, \mathcal{V}, \mathcal{K}, \mathcal{K}^{\prime}, \mathcal{R}\right)$ is a tuple (SetupSound, SetupABO, Sample, Prove, SimProve, Verify, PubEval, SharePrivEval, ShareEvalVerify, Combine) of efficient algorithms with the following specifications.

SetupSound $(\lambda, t, n)$ : given a security parameter $\lambda \in \mathbb{N}$ and integers $t, n \in \operatorname{poly}(\lambda)$, this algorithm outputs a public key pk, $n$ private key shares $\left(\mathrm{sk}_{1}, \ldots, \mathrm{sk}_{n}\right)$ and verification keys $\left(\mathrm{vk}_{1}, \ldots, \mathrm{vk}_{n}\right)$.

$\operatorname{SetupABO}\left(\lambda, t, n, \operatorname{tag}^{\star}\right)$ : takes as input a security parameter $\lambda \in \mathbb{N}$, integers $t, n \in \operatorname{poly}(\lambda)$ and a tag tag*. It outputs a public key pk, $n$ private key shares $\left(\mathrm{sk}_{1}, \ldots, \mathrm{sk}_{n}\right)$, the corresponding verification keys $\left(\mathrm{vk}_{1}, \ldots, \mathrm{vk}_{n}\right)$ as well as a simulation trapdoor $\tau$. It is important that $\tau$ be independent of $\left\{\mathrm{sk}_{i}\right\}_{i=1}^{n}$.

Sample(pk): is a probabilistic algorithm that takes as input a public key pk. It draws random coins $r \stackrel{R}{-} \mathcal{R}$ and outputs an element $\Phi \in \mathcal{V}$ along with the random coins $r$ that will serve as a witness explaining $\Phi$ as an element of $\mathcal{V}$.

Prove (pk, tag, $r, \Phi)$ : takes in a public key pk, a tag tag, an element $\Phi \in \mathcal{V}$ and the random coins $r \in \mathcal{R}$ that were used to sample $\Phi$. It generates a non-interactive proof $\pi_{\mathcal{V}}$ that $\Phi \in \mathcal{V}$.

SimProve $(\mathrm{pk}, \tau, \operatorname{tag}, \Phi)$ : takes as input a public key pk and a simulation trapdoor $\tau$ produced by $\operatorname{SetupABO}\left(\lambda, t, n, \operatorname{tag}^{\star}\right)$, a tag tag and an element $\Phi \in \mathcal{C}$. If $\operatorname{tag} \neq \operatorname{tag}^{\star}$, the algorithm outputs $\perp$. If $\operatorname{tag}=\operatorname{tag}^{\star}$, the algorithm produces a simulated NIZK proof $\pi_{\mathcal{V}}$ that $\Phi \in \mathcal{V}$.

Verify $\left(\mathrm{pk}, \operatorname{tag}, \Phi, \pi_{\mathcal{V}}\right):$ takes as input a public key pk, a tag tag, an element $\Phi \in \mathcal{C}$ and a purported proof $\pi_{\mathcal{V}}$. It outputs 1 if and only if $\pi_{\mathcal{V}}$ is deemed as a valid proof that $\Phi \in \mathcal{V} \subset \mathcal{C}$.

PubEval(pk, $r, \Phi)$ : takes as input a public key pk, an element $\Phi \in \mathcal{V}$ and the random coins $r \in_{R} \mathcal{R}$ such that $(r, \Phi) \leftarrow$ Sample(pk). It outputs a value $K \in \mathcal{K}$, which is called public evaluation of $\Phi$.

SharePrivEval $\left(\mathrm{pk}_{,} \mathrm{sk}_{i}, \Phi\right)$ : is a deterministic algorithm that takes in a public key pk, a private key share $\mathrm{sk}_{i}$ and an element $\Phi \in \mathcal{C}$. It outputs a value $K_{i} \in \mathcal{K}^{\prime}$, called private evaluation share and a proof $\pi_{K_{i}}$ that $K_{i}$ was evaluated correctly.

ShareEvalVerify $\left(\mathrm{pk}, \mathrm{vk}_{i}, \Phi, K_{i}, \pi_{K_{i}}\right)$ : given a public key pk, a verification key $\mathrm{vk}_{i}$, an element $\Phi \in \mathcal{C}$, a private evaluation share $K_{i} \in \mathcal{K}^{\prime}$ and its proof $\pi_{K_{i}}$, this algorithm outputs 1 if $\pi_{K_{i}}$ is considered as a valid proof of the correct evaluation of $K_{i}$. Otherwise, it outputs 0 .

Combine(pk, $\left.\Phi,\left\{\left(K_{i}, \pi_{K_{i}}\right)\right\}_{i \in S}\right)$ : takes as input a public key pk, an element $\Phi \in \mathcal{C}$ and a set of $t$ pairs $\left\{\left(K_{i}, \pi_{K_{i}}\right)\right\}_{i \in S}$, where $S \subset\{1, \ldots, n\}$, each one of which consists of a private evaluation share $K_{i} \in \mathcal{K}^{\prime}$ and its proof $\pi_{K_{i}}$. If ShareEvalVerify $\left(\mathrm{pk}, \mathrm{vk}_{i}, \Phi, K_{i}, \pi_{K_{i}}\right)=0$ for some $i \in S$, it outputs $\perp$. Otherwise, it outputs a value $K \in \mathcal{K}$. 
We also define this algorithm which is implied by the above ones but will be convenient to use.

PrivEval(pk, $\left.\left\{\mathrm{sk}_{i}\right\}_{i \in S}, \Phi\right)$ : given a public key pk, a set of private key shares $\left\{\mathbf{s k}_{i}\right\}_{i \in S}$ where $S$ is an arbitrary $t$-subset of $\{1, \ldots, n\}$, and an element $\Phi \in \mathcal{C}$, this algorithm outputs the result of Combine $\left(\mathrm{pk}, \Phi,\left\{\left(K_{i}, \pi_{K_{i}}\right)\right\}_{i \in S}\right)$ where $\left(K_{i}, \pi_{K_{i}}\right) \leftarrow$ SharePrivEval $\left(\mathrm{pk}, \mathrm{sk}_{i}, \Phi\right)$ for each $i \in S$.

The following properties are required from these algorithms and the sets $\left(\mathcal{C}, \mathcal{V}, \mathcal{K}, \mathcal{K}^{\prime}, \mathcal{R}\right)$.

(Setup Indistinguishability): For any integers $(\lambda, t, n)$ such that $1 \leq t \leq n$ and any tag tag ${ }^{\star}$, the output of SetupSound $(\lambda, t, n)$ and the outputs (pk, $\left.\left\{\mathrm{sk}_{i}\right\}_{i=1}^{n},\left\{\mathrm{vk}_{i}\right\}_{i=1}^{n}\right)$ of $\operatorname{SetupABO}\left(\lambda, t, n, \operatorname{tag}^{\star}\right)$ are computationally indistinguishable. For any integers $(\lambda, t, n)$ such that $1 \leq t \leq n$, any tag $\operatorname{tag}^{\star}$, and any PPT distinguisher $\mathcal{D}$, we must have $\operatorname{Adv}_{\mathcal{D}}^{\text {Setup-indist }}(\lambda) \in \operatorname{negl}(\lambda)$ when the latter advantage function is defined as the distance

$$
\begin{aligned}
\mid \operatorname{Pr} & {\left[\mathcal{D}\left(\mathrm{pk},\left\{\mathrm{sk}_{i}\right\}_{i=1}^{n},\left\{\mathrm{vk}_{i}\right\}_{i=1}^{n}\right)=1 \mid\left(\mathrm{pk},\left\{\mathrm{sk}_{i}\right\}_{i=1}^{n},\left\{\mathrm{vk}_{i}\right\}_{i=1}^{n}\right) \leftarrow \operatorname{SetupSound}(\lambda, t, n)\right] } \\
& -\operatorname{Pr}\left[\mathcal{D}\left(\mathrm{pk},\left\{\mathbf{s k}_{i}\right\}_{i=1}^{n},\left\{\mathrm{vk}_{i}\right\}_{i=1}^{n}\right)=1 \mid\left(\mathrm{pk},\left\{\mathbf{s k}_{i}\right\}_{i=1}^{n},\left\{\mathrm{vk}_{i}\right\}_{i=1}^{n}\right) \leftarrow \operatorname{SetupABO}\left(\lambda, t, n, \operatorname{tag}^{\star}\right)\right] \mid
\end{aligned}
$$

(Correctness and Public Evaluability on $\mathcal{V}$ ): For any (pk, $\left\{\mathbf{s k}_{i}\right\}_{i=1}^{n},\left\{\mathbf{v k}_{i}\right\}_{i=1}^{n}$ ) returned by SetupSound or SetupABO, if $(r, \Phi) \stackrel{R}{\leftarrow}$ Sample(pk) (and thus $\Phi \in \mathcal{V}$ ), it holds that:

1. For any $i \in\{1, \ldots, n\}$, if $\left(K_{i}, \pi_{K_{i}}\right) \leftarrow$ SharePrivEval $\left(\mathrm{pk} \mathrm{sk}_{i}, \Phi\right)$, then the private evaluation share $K_{i} \in \mathcal{K}^{\prime}$ is uniquely determined by $\left(\mathrm{pk}, \mathrm{vk}_{i}\right)$ and $\Phi$. Moreover, the proof $\pi_{K_{i}}$ satisfies the verification test: ShareEvalVerify $\left(\mathrm{pk}, \mathrm{vk}_{i}, \Phi, K_{i}, \pi_{K_{i}}\right)=1$.

2. For any $t$-subset $S \subset\{1, \ldots, n\}$, combining the corresponding private evaluation shares allows recomputing the public evaluation:

$$
\operatorname{PubEval}(\text { pk, } r, \Phi)=\operatorname{PrivEval}\left(\text { pk },\left\{\mathrm{sk}_{i}\right\}_{i \in S}, \Phi\right) .
$$

(Universality): For any (pk, $\left.\left\{\mathbf{s k}_{i}\right\}_{i=1}^{n},\left\{\mathbf{v k}_{i}\right\}_{i=1}^{n}\right)$ produced by SetupSound or SetupABO and any $\Phi \in \mathcal{C} \backslash \mathcal{V}$, for any subset $\bar{S} \subset\{1, \ldots, n\}$ of size $|\bar{S}|=t-1$, the statistical distance

$$
\Delta\left[\left(\mathrm{pk},\left\{\mathrm{vk}_{i}\right\}_{i=1}^{n},\left\{\mathrm{sk}_{i}\right\}_{i \in \bar{S}}, \Phi, \operatorname{PrivEval}\left(\mathrm{pk},\left\{\mathrm{sk}_{i}\right\}_{i=1}^{t}, \Phi\right)\right),\left(\mathrm{pk},\left\{\mathrm{vk}_{i}\right\}_{i=1}^{n},\left\{\mathrm{sk}_{i}\right\}_{i \in \bar{S}}, \Phi, K\right)\right],
$$

where $K \stackrel{R}{\leftarrow} \mathcal{K}$, should be smaller than $2^{-\lambda}$.

(All-But-One Soundness): For all integers $(\lambda, t, n)$ such that $1 \leq t \leq n$, any tag tag ${ }^{\star}$ and any outputs (pk, $\left.\left\{\mathrm{sk}_{i}\right\}_{i=1}^{n},\left\{\mathrm{vk}_{i}\right\}_{i=1}^{n}, \tau\right)$ of $\operatorname{SetupABO}\left(\lambda, t, n, \operatorname{tag}^{\star}\right)$, these conditions are satisfied.

1. For any tag $\neq \operatorname{tag}^{\star}$, proofs are always perfectly sound. Namely, if a proof $\pi_{\mathcal{V}}$ satisfies $\operatorname{Verify}\left(\mathrm{pk}, \operatorname{tag}, \Phi, \pi_{\mathcal{V}}\right)=1$ for some $\Phi \in \mathcal{C}$, then it necessarily holds that $\Phi \in \mathcal{V}$.

2. For any $\Phi \in \mathcal{C}$, the trapdoor $\tau$ allows simulating a proof $\pi_{\mathcal{V}} \leftarrow \operatorname{SimProve}\left(\mathrm{pk}, \tau, \operatorname{tag}^{\star}, \Phi\right)$ such that $\operatorname{Verify}\left(\mathrm{pk}, \operatorname{tag}^{\star}, \Phi, \pi_{\mathcal{V}}\right)=1$ (note that $\pi_{\mathcal{V}}$ is a proof for a false statement if $\Phi \in \mathcal{C} \backslash \mathcal{V}$ ). Moreover, if $\Phi \in \mathcal{V}$, the simulated proof $\pi_{\mathcal{V}}$ should be perfectly indistinguishable from a real proof (i.e., that would be generated by Prove using a witness $r \in \mathcal{R}$ of the fact that $\Phi \in \mathcal{V}$ ). 
(Simulatability of Share Proofs): For all integers $(\lambda, t, n)$ such that $1 \leq t \leq n$, any tag tag ${ }^{\star}$, any outputs (pk, $\left.\left\{\mathrm{sk}_{i}\right\}_{i=1}^{n},\left\{\mathrm{vk}_{i}\right\}_{i=1}^{n}, \tau\right)$ of $\operatorname{SetupABO}\left(\lambda, t, n, \operatorname{tag}^{\star}\right)$ and any $\Phi \in \mathcal{C}$, the proofs $\pi_{K_{i}}$ produced by $\left(K_{i}, \pi_{K_{i}}\right) \leftarrow$ SharePrivEval $\left(\mathrm{pk}, \mathrm{sk}_{i}, \Phi\right)$ should be simulatable using the trapdoor $\tau$ instead of $\left\{\mathbf{s k}_{i}\right\}_{i=1}^{n}$. Using $\tau$ and public values (pk, $\left.\left\{\mathrm{vk}_{i}\right\}_{i=1}^{n}, \Phi\right)$, an efficient algorithm $\mathcal{S}$ should be able to produce simulated proofs $\pi_{K_{i}}$ that are perfectly indistinguishable from real proofs.

(Consistency): For all integers $(\lambda, t, n)$ such that $1 \leq t \leq n$, any output (pk, $\left.\left\{\left(\mathrm{vk}_{i}, \mathrm{sk}_{i}\right)\right\}_{i=1}^{n}\right)$ of SetupSound $(\lambda, t, n)$, given ( $\left.\mathrm{pk},\left\{\left(\mathrm{vk}_{i}, \mathrm{sk}_{i}\right)\right\}_{i=1}^{n}\right)$, it should be computationally infeasible to come up with a triple $\left(\operatorname{tag}, \Phi, \pi_{\mathcal{V}}\right)$ as well as two distinct $t$-sets $\Gamma=\left\{\left(K_{i_{1}}, \pi_{K_{i_{1}}}\right), \ldots,\left(K_{i_{t}}, \pi_{K_{i_{t}}}\right)\right\}$ and $\Gamma^{\prime}=\left\{\left(K_{j_{1}}^{\prime}, \pi_{K_{j_{1}}}^{\prime}\right), \ldots,\left(K_{j_{t}}^{\prime}, \pi_{K_{j_{t}}}^{\prime}\right)\right\}$, with $i_{k}, j_{k} \in\{1, \ldots, n\}$ for each $k \in\{1, \ldots, t\}$, such that: (i) Verify (pk, tag, $\left.\Phi, \pi_{\mathcal{V}}\right)=1$; (ii) for each $k \in\{1, \ldots, t\}$, ShareEvalVerify $\left(\mathrm{pk}, \mathrm{vk}_{i_{k}}, \Phi, K_{i_{k}}, \pi_{K_{i_{k}}}\right)=1$ and ShareEvalVerify $\left(\mathrm{pk}, \mathrm{vk}_{j_{k}}, \Phi, K_{j_{k}}^{\prime}, \pi_{K_{j_{k}}}^{\prime}\right)=1$; (iii) Combine(pk, $\left.\Phi, \Gamma\right) \neq \operatorname{Combine}\left(\mathrm{pk}, \Phi, \Gamma^{\prime}\right)$.

(Subset Membership Hardness): membership in $\mathcal{C}$ should be easy to check but membership in $\mathcal{V}$ should not. Moreover, this should hold even if $\tau$ is given. Namely, for all integers $(\lambda, t, n)$ such that $1 \leq t \leq n$, any tag tag ${ }^{\star}$ and any outputs (pk, $\left.\left\{\mathrm{sk}_{i}\right\}_{i=1}^{n},\left\{\mathrm{vk}_{i}\right\}_{i=1}^{n}, \tau\right)$ of $\operatorname{SetupABO}\left(\lambda, t, n, \operatorname{tag}^{\star}\right)$, for any PPT distinguisher $\mathcal{D}$, it must hold that:

$$
\begin{aligned}
\operatorname{Adv}_{\mathcal{D}}^{\mathrm{SM}}(\lambda)=\mid \operatorname{Pr}\left[\mathcal{D}\left(\mathrm{pk},\left\{\left(\mathrm{sk}_{i}, \mathrm{vk}_{i}\right)\right\}_{i=1}^{n}, \mathcal{C}, \mathcal{V}, C_{1}, \tau\right)=1 \mid C_{1} \stackrel{R}{-} \mathcal{C} \backslash \mathcal{V}\right] \\
\quad-\operatorname{Pr}\left[\mathcal{D}\left(\mathrm{pk},\left\{\left(\mathrm{sk}_{i}, \mathrm{vk}_{i}\right)\right\}_{i=1}^{n}, \mathcal{C}, \mathcal{V}, C_{0}, \tau\right)=1 \mid C_{0} \stackrel{R}{\leftarrow} \mathcal{V}\right] \mid \in \operatorname{negl}(\lambda) .
\end{aligned}
$$

In the definition of the subset membership hardness property, the trapdoor $\tau$ should not carry any side information helping the distinguisher. For this reason, the latter receives $\tau$ as part of its input.

Looking ahead, the security proof of our threshold cryptosystems will rely on the Subset Membership hardness property to reach a game where the plaintext is statistically independent of the adversary's view. In the final transition, the reduction must be able to simulate a possibly fake proof that the ciphertext is valid (i.e., a proof that some ciphertext component $C$ lives in $\mathcal{V}$ although it could have been sampled from $\mathcal{C} \backslash \mathcal{V})$ using the trapdoor $\tau$.

\section{Adaptively Secure Robust Non-Interactive CCA2-Secure Threshold Cryptosystems from All-But-One Perfectly Sound Threshold Hash Proof Systems}

Let $\Pi^{\text {ABO-THPS }}=($ SetupSound, SetupABO, Sample, Prove, SimProve, Verify, PubEval, SharePrivEval, ShareEvalVerify, Combine) be an all-but-one perfectly sound threshold hash proof system for sets $\left(\mathcal{C}, \mathcal{V}, \mathcal{K}, \mathcal{K}^{\prime}, \mathcal{R}\right)$ that satisfy the conditions specified in Section 3 . We assume that messages are in $\mathcal{K}$. The generic construction of CCA2-secure threshold cryptosystem goes as follows.

$\operatorname{Keygen}(\lambda, t, n)$ : given integers $\lambda, t, n \in \mathbb{N}$, choose a one-time signature scheme $\Sigma=$ (Gen, Sig, Ver), generate $\left(\mathrm{pk},\left\{\mathbf{s k}_{i}\right\}_{i=1}^{n},\left\{\mathrm{vk}_{i}\right\}_{i=1}^{n}\right) \leftarrow \operatorname{SetupSound}(\lambda, t, n)$ and output $(P K, \mathbf{S K}, \mathbf{V K})$, where the vectors of private key shares and verification keys are defined as $\mathbf{S K}=\left(\mathbf{s k}_{1}, \ldots, \mathbf{s k}_{n}\right)$ and $\mathbf{V K}=\left(\mathrm{vk}_{1}, \ldots, \mathrm{vk}_{n}\right)$, respectively. The public key is $P K=(\mathrm{pk}, \Sigma)$.

$\operatorname{Encrypt}(M, P K)$ : to encrypt a message $M \in \mathcal{K}$ using $P K=(\mathrm{pk}, \Sigma)$,

1. Generate a one-time signature key pair $(\mathrm{SSK}, \mathrm{SVK}) \leftarrow \Sigma$.Gen $(\lambda)$.

2. Choose $r \stackrel{R}{\leftarrow} \mathcal{R}$, compute $(r, \Phi) \leftarrow$ Sample $(\mathrm{pk}, r)$ as well as $C_{0}=M \odot \operatorname{PubEval}(\mathrm{pk}, r, \Phi)$.

3. Generate a proof $\pi_{\mathcal{V}} \leftarrow$ Prove $(\mathrm{pk}, \mathrm{SVK}, r, \Phi)$ that $\Phi \in \mathcal{V}$ with respect to the tag SVK. 
4. Output the ciphertext $C=\left(\mathrm{SVK}, C_{0}, \Phi, \pi_{\mathcal{V}}, \sigma\right)$, where $\sigma=\Sigma \cdot \operatorname{Sig}\left(\mathrm{SSK},\left(C_{0}, \Phi, \pi_{\mathcal{V}}\right)\right)$.

Ciphertext-Verify $(P K, C)$ : parse $C$ as $C=\left(\mathrm{SVK}, C_{0}, \Phi, \pi_{\mathcal{V}}, \sigma\right)$ and $P K$ as (pk, $\left.\Sigma\right)$. Return 1 if $\Sigma . \operatorname{Ver}\left(\mathrm{SVK},\left(C_{0}, \Phi, \pi_{\mathcal{V}}\right), \sigma\right)=1$ and Verify $\left(\mathrm{pk}, \mathrm{SVK}, \Phi, \pi_{\mathcal{V}}\right)=1$. Otherwise, return 0.

Share-Decrypt $\left(S K_{i}, C\right)$ : given the private key share $S K_{i}=\mathrm{sk}_{i}$ and $C=\left(\mathrm{SVK}, C_{0}, \Phi, \pi_{\mathcal{V}}, \sigma\right)$, return $(i, \perp)$ if it turns out that Ciphertext-Verify $(P K, C)=0$. Otherwise, compute a pair $\left(K_{i}, \pi_{K_{i}}\right) \leftarrow$ SharePrivEval $\left(\mathrm{pk}, \mathrm{sk}_{i}, \Phi\right)$ and return $\mu_{i}=\left(i, \hat{\mu}_{i}\right)$ where $\hat{\mu}_{i}=\left(K_{i}, \pi_{K_{i}}\right)$.

Share-Verify $\left(P K, V K_{i}, C,\left(i, \hat{\mu}_{i}\right)\right)$ : parse the ciphertext $C$ as $\left(\mathrm{SVK}, C_{0}, \Phi, \pi_{\mathcal{V}}, \sigma\right)$. If the decryption share $\hat{\mu}_{i}$ is such that $\hat{\mu_{i}}=\perp$ or if it cannot be properly parsed as a pair $\left(K_{i}, \pi_{K_{i}}\right)$, return 0 . Otherwise, return 1 if ShareEvalVerify $\left(\mathrm{pk}, \mathrm{vk}_{i}, \Phi, K_{i}, \pi_{K_{i}}\right)=1$. In any other situation, return 0 .

Combine $\left(P K, \mathbf{V K}, C,\left\{\left(i, \hat{\mu_{i}}\right)\right\}_{i \in S}\right)$ : parse $C$ as $\left(\mathrm{SVK}, C_{0}, \Phi, \pi_{\mathcal{V}}, \sigma\right)$. Return $\perp$ if there exists $i \in S$ such that Share-Verify $\left(P K, C,\left(i, \hat{\mu}_{i}\right)\right)=0$ or if Ciphertext-Verify $(P K, C)=0$. Otherwise, compute $K=$ Combine $\left(\mathrm{pk}, \Phi,\left\{\left(K_{i}, \pi_{K_{i}}\right)\right\}_{i \in S}\right) \in \mathcal{K}$, which unveils $M=C_{0} \odot K^{-1}$.

We observe that there is no need to bind the one-time verification key SVK to the ciphertext components $\left(C_{0}, \Phi, \pi_{\mathcal{V}}\right)$ in any other way than by using it as a tag to compute the non-interactive proof $\pi_{\mathcal{V}}$. Indeed, if the adversary attempts to re-use parts $\left(C_{0}^{\star}, \Phi^{\star}, \pi_{\mathcal{V}}^{\star}\right)$ of the challenge ciphertext and simply replaces the one-time verification key SVK ${ }^{\star}$ by a verification key SVK of its own, it will be forced to compute a proof $\pi_{\mathcal{V}}$ that correspond to the same $\Phi^{\star}$ as in the challenge phase but under the new tag SVK. Our security proof shows that this is infeasible as long as $\Pi^{\mathrm{ABO}-\mathrm{THPS}}$ satisfies the properties of setup indistinguishability and all-but-one soundness.

The consistency property of the scheme is trivially implied by that of $\Pi^{\text {ABO-THPS }}$ and we focus on proving its IND-CCA security. In the threshold setting, adaptive security is achieved by taking advantage of the fact that, in security reductions using hash proof systems, the simulator typically knows the private key and can thus answer adaptive queries at will. At the same time, invalid ciphertexts are harmless as they are made publicly recognizable due to the use of non-interactive proofs of validity: as long as these proofs are perfectly sound in all decryption queries, the simulator is guaranteed not to leak too much information about the particular private key it is using.

The main problem to solve is thus to make sure that only the simulator can simulate a fake proof in the challenge phase, and this is where the all-but-one soundness property is handy.

Theorem 1. The above threshold cryptosystem is IND-CCA secure against adaptive corruptions assuming that: (i) $\Pi^{\mathrm{ABO}-\mathrm{THPS}}$ is an all-but-one perfectly sound hash proof system; (ii) $\Sigma$ is a strongly unforgeable one-time signature. Namely, for any adversary $\mathcal{A}$ making $Q$ decryption queries within running time $t$, there exist adversaries $\mathcal{D}_{1}, \mathcal{D}_{2}$ and $\mathcal{D}_{2}$ running in time $t^{\prime} \approx t+Q \cdot \operatorname{poly}(\lambda)$ such that

$$
\mathbf{A d v}(\mathcal{A}) \leq \mathbf{A d v}_{\mathcal{D}_{1}}^{\text {Setup-indist }}(\lambda)+\mathbf{A d v}_{\mathcal{D}_{2}}^{\mathrm{OTS}}(\lambda)+\mathbf{A d v}_{\mathcal{D}_{3}}^{\mathrm{SM}}(\lambda)+\frac{1}{2^{\lambda}}
$$

Proof. The proof uses of a sequence of games starting with the real attack game and ending with a game where the adversary $\mathcal{A}$ has no advantage. For each $i, S_{i}$ is the event that $\mathcal{A}$ wins in Game .

Game $_{1}$ : is the real attack game. In details, the adversary is given the public key $P K$ and the set of verification keys $\mathbf{V K}=\left(\mathrm{vk}_{1}, \ldots, \mathrm{vk}_{n}\right)$ and starts making adaptive queries. At each corruption query $i \in\{1, \ldots, n\}$, the challenger $\mathcal{B}$ reveals the queried private key share $S K_{i}=$ sk $_{i}$ and, at 
each decryption query, $\mathcal{B}$ runs the real shared decryption algorithm. In the challenge phase, the adversary $\mathcal{A}$ chooses messages $M_{0}, M_{1} \in \mathcal{K}$ and obtains $C^{\star}=\left(\mathrm{SVK}^{\star}, C_{0}^{\star}, \Phi^{\star}, \pi_{\mathcal{V}}^{\star}, \sigma^{\star}\right)$ which is an encryption of $M_{\beta}$, for some random coin $\beta \stackrel{R}{\leftarrow}\{0,1\}$ internally flipped by $\mathcal{B}$. For simplicity, we assume that the one-time signature key pair $\left(\mathrm{SSK}^{\star}, \mathrm{SVK}^{\star}\right)$ is chosen by $\mathcal{B}$ at the outset of the game. In the second phase, $\mathcal{A}$ makes more adaptive queries under the restriction of not asking for a partial decryption of $C^{\star}$ or for more than $t-1$ private key shares throughout the entire game. Eventually, $\mathcal{A}$ halts and outputs $\beta^{\prime}$. We denote by $S_{1}$ the event that $\beta=\beta^{\prime}$.

Game $_{2}$ : We change the distribution of the public key $P K=(\mathrm{pk}, \Sigma)$. Namely, instead of generating (pk, $\left.\left\{\mathbf{s k}_{i}\right\}_{i=1}^{n},\left\{\mathbf{v k}_{i}\right\}_{i=1}^{n}\right)$ as per $\left(\mathrm{pk},\left\{\mathbf{s k}_{i}\right\}_{i=1}^{n},\left\{\mathbf{v k}_{i}\right\}_{i=1}^{n}\right) \leftarrow$ SetupSound $(\lambda, t, n)$, the challenger $\mathcal{B}$ runs the all-but-one setup algorithm $\left(\mathrm{pk},\left\{\mathrm{sk}_{i}\right\}_{i=1}^{n},\left\{\mathrm{vk}_{i}\right\}_{i=1}^{n}, \tau\right) \leftarrow \operatorname{SetupABO}\left(\lambda, t, n, \mathrm{SVK}^{\star}\right)$, discards $\tau$ and uses (pk, $\left.\left\{\mathbf{s k}_{i}\right\}_{i=1}^{n},\left\{\mathbf{v k}_{i}\right\}_{i=1}^{n}\right)$ as in Game ${ }_{1}$. We note that, after this change, the one-time verification key $\mathrm{SVK}^{\star}$ may not be statistically independent of $\mathcal{A}$ 's view before the challenge phase. However, due to the setup indistinguishability property of $\Pi^{\text {ABO-THPS }}$, this modification cannot significantly affect $\mathcal{A}$ 's behavior. A straightforward reduction from the setup indistinguishability property shows that $\left|\operatorname{Pr}\left[S_{2}\right]-\operatorname{Pr}\left[S_{1}\right]\right| \leq \mathbf{A d v}_{\mathcal{D}}^{\text {Setup-indist }}(\lambda)$.

Game 3 : We introduce a failure event $F_{3}$ and let the challenger $\mathcal{B}$ halt and output a random bit if $F_{3}$ occurs. We call $F_{3}$ the event that $\mathcal{A}$ makes a decryption query involving a valid ciphertext $C=\left(\mathrm{SVK}, C_{0}, \Phi, \pi_{\mathcal{V}}, \sigma\right)$ such that $\mathrm{SVK}=\mathrm{SVK}^{\star}$. We note that Game 3 and $\mathrm{Game}_{2}$ are identical until $F_{3}$ occurs, so that $\left|\operatorname{Pr}\left[S_{3}\right]-\operatorname{Pr}\left[S_{2}\right]\right| \leq \operatorname{Pr}\left[F_{3}\right]$. Moreover, we argue that event $F_{3}$ would defeat the strong unforgeability of the one-time signature, so that $\operatorname{Pr}\left[F_{3}\right] \leq \mathbf{A d v}^{\mathrm{OTS}}(\lambda)$. Indeed, if $F_{3}$ occurs before the challenge phase, $\mathcal{A}$ immediately implies a forger that breaks the standard unforgeability of the one-time signature without making any signing query. If $F_{3}$ comes about in a post-challenge decryption query, $\mathcal{A}$ yields a forger that breaks the strong unforgeability of the one-time signature. (The details of the reduction are straightforward and omitted.)

$\mathrm{Game}_{4}$ : We modify the generation of the challenge ciphertext $C^{\star}$. Namely, the challenger still picks $\Phi^{\star} \in \mathcal{V}$ as per $\left(r^{\star}, \Phi^{\star}\right) \leftarrow$ Sample(pk), using random coins $r^{\star} \stackrel{R}{\leftarrow} \mathcal{R}$, as in previous games. However, $C_{0}^{\star}$ is now computed as $C_{0}^{\star}=M_{\beta} \odot \operatorname{PrivEval}\left(\right.$ pk, $\left.\left\{\text { sk }_{i}\right\}_{i=1}^{t}, \Phi^{\star}\right)$ (instead of $C_{0}^{\star}=M_{\beta} \odot$ PubEval $\left.\left(\mathrm{pk}, r^{\star}, \Phi^{\star}\right)\right)$. As long as $\Pi^{\mathrm{ABO}-T H P S}$ satisfies the property of correctness and public evaluability on $\mathcal{V}, \mathcal{A}$ 's view does not change since $C_{0}^{\star}$ has the same distribution either way. We thus have $\operatorname{Pr}\left[S_{4}\right]=\operatorname{Pr}\left[S_{3}\right]$.

Game $_{5}$ : We modify again the generation of the challenge ciphertext $C^{\star}$. We observe that the proof $\pi_{\mathcal{V}}^{\star}$ must be generated w.r.t. the tag $\mathrm{SVK}^{\star}$ which, due to the modification introduced in Game 2 , is the tag for which $\mathcal{B}$ can generate simulated NIZK proofs using the trapdoor $\tau$. To construct the ciphertext $C^{\star}$, the challenger $\mathcal{B}$ chooses $\Phi^{\star} \in \mathcal{V}$ as in $\mathrm{Game}_{4}$ and sets

$$
C_{0}^{\star}=M_{\beta} \odot \operatorname{PrivEval}\left(\mathrm{pk},\left\{\mathrm{sk}_{i}\right\}_{i=1}^{t}, \Phi^{\star}\right), \quad \pi_{\mathcal{V}}^{\star}=\operatorname{SimProve}\left(\mathrm{pk}, \tau, \mathrm{SVK}^{\star}, \Phi^{\star}\right) .
$$

Note that, with this modification, $\pi_{\mathcal{V}}^{\star}$ is now generated using $\tau$ instead of $\left\{\mathbf{s k}_{i}\right\}_{i=1}^{n}$. Since $\tau$ is independent of $\left\{\mathbf{s k}_{i}\right\}_{i=1}^{n}$, it does not reveal any more information about $\left\{\mathbf{s k}_{i}\right\}_{i=1}^{n}$ than pk and $\left\{\mathrm{vk}_{i}\right\}_{i=1}^{n}$. Since $\left(C_{0}^{\star}, \Phi^{\star}, \pi_{\mathcal{V}}^{\star}\right)$ have the same distribution as in $\mathrm{Game}_{4}$, we have $\operatorname{Pr}\left[S_{5}\right]=\operatorname{Pr}\left[S_{4}\right]$.

Game $_{6}$ : is as Game ${ }_{5}$ but we change the treatment of decryption queries $C=\left(\mathrm{SVK}, C_{0}, \Phi, \pi_{\mathcal{V}}, \sigma\right)$. More precisely, whenever $\mathcal{B}$ runs SharePrivEval $\left(\mathrm{pk}, \mathrm{sk}_{i}, \Phi\right)$ in order to answer decryption queries, to obtain a private evaluation share $K_{i}$ and a proof $\pi_{K_{i}}$ of its validity, the latter is generated 
using the simulator $\mathcal{S}$ and the simulation trapdoor $\tau$ instead of the private key share $\mathbf{s k}_{i}$. The property that we called "simulatability of share proofs" guarantees the existence of such an efficient simulator $\mathcal{S}$ and that simulated proofs $\pi_{K_{i}}$ will be distributed as real proofs. Hence, we can write $\operatorname{Pr}\left[S_{6}\right]=\operatorname{Pr}\left[S_{5}\right]$.

Game $_{7}$ : We bring one last change in the generation of the challenge ciphertext. Instead of computing $\left(C_{0}^{\star}, \pi_{\mathcal{V}}^{\star}\right)$ as per (2) using a random $\Phi^{\star} \in \mathcal{V}$, the value $\Phi^{\star}$ is randomly chosen in $\mathcal{C} \backslash \mathcal{V}$. Under the subset membership hardness assumption in $(\mathcal{C}, \mathcal{V})$, this modification cannot be noticed by $\mathcal{A}$.

The reduction $\mathcal{B}$ proceeds in the following way. It takes as input a Subset Membership instance $(\mathcal{C}, \mathcal{V}, C, \tau)$ for which it must decide if $C \in_{R} \mathcal{C} \backslash \mathcal{V}$ or $C \in_{R} \mathcal{V}$. To this end, it generates the public key and answers all decryption queries exactly as in $\mathrm{Game}_{6}$. In the challenge phase, $\mathcal{B}$ constructs the challenge ciphertext by defining $\Phi^{\star}=C$ and computing $\left(C_{0}^{\star}, \pi_{\mathcal{V}}^{\star}\right)$ as per $(2)$. In particular, the trapdoor $\tau$, which is part of the Subset Membership instance, is used to simulate $\pi_{\mathcal{V}}^{\star}$. If $C \in_{R} \mathcal{V}$, the adversary's view is identical to that of Game ${ }_{6}$. In contrast, if $C \in_{R} \mathcal{C} \backslash \mathcal{V}$, the challenge ciphertext $C^{\star}=\left(\mathrm{SVK}^{\star}, C_{0}^{\star}, \Phi^{\star}, \pi_{\mathcal{V}}^{\star}, \sigma^{\star}\right)$ is distributed as in Game ${ }_{7}$. Consequently, we must have $\left|\operatorname{Pr}\left[S_{7}\right]-\operatorname{Pr}\left[S_{6}\right]\right| \leq \mathbf{A d v}_{\mathcal{A}}^{\mathrm{SM}}(\lambda)$ for any PPT adversary $\mathcal{A}$.

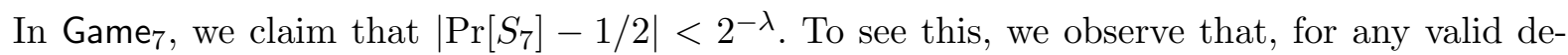
cryption query $C=\left(\mathrm{SVK}, C_{0}, \Phi, \pi_{\mathcal{V}}, \sigma\right)$ such that $\mathrm{SVK} \neq \mathrm{SVK}^{\star}$, the proof $\pi_{\mathcal{V}}$ is perfectly sound since it is generated for a tag $\mathrm{SVK} \neq \mathrm{SVK}^{\star}$ that ensures perfect soundness. This guarantees that $\Phi \in \mathcal{V}$ (as even an unbounded $\mathcal{A}$ would be unable to generate a convincing proof $\pi_{\mathcal{V}}$ otherwise). Consequently, for each revealed decryption share $\hat{\mu}_{i}=\left(i,\left(K_{i}, \pi_{K_{i}}\right)\right)$, it holds that: (1) $K_{i}$ does not reveal any more information about $\mathrm{sk}_{i}$ than $\left(\mathrm{pk}, \mathrm{vk}_{i}\right)$ since it is uniquely determined by $\left(\mathrm{pk}, \mathrm{vk}_{i}, \Phi\right)$; (2) the distribution of $\pi_{K_{i}}$ does not depend on $\mathrm{sk}_{i}$ thanks to the modification introduced in Game ${ }_{6}$.

The universality property of $\Pi^{\mathrm{ABO}-T H P S}$ tells us that, for any adaptively-chosen $(t-1)$-subset $\bar{S} \subset\{1, \ldots, n\}$, the distribution (pk, $\left\{\mathrm{vk}_{i}\right\}_{i=1}^{n},\left\{\mathrm{sk}_{i}\right\}_{i \in \bar{S}}, \Phi^{\star}, \operatorname{PrivEval}\left(\mathrm{pk},\left\{\mathrm{sk}_{i}\right\}_{i=1}^{t}, \Phi^{\star}\right)$ ) is with statistical distance $2^{-\lambda}$ from the distribution $\left(\mathrm{pk},\left\{\mathrm{vk}_{i}\right\}_{i=1}^{n},\left\{\mathrm{sk}_{i}\right\}_{i \in \bar{S}}, \Phi^{\star}, K\right)$, where $K \stackrel{R}{\leftarrow} \mathcal{K}$. In other words, $C_{0}^{\star}$ statistically hides $M_{\beta}$ and $\operatorname{Pr}\left[S_{7}\right]$ is within distance $2^{-\lambda}$ from $1 / 2$, as claimed. The stated upper bound (1) follows by counting probabilities throughout the sequence of games.

We remark that the security bound (1) is tight in that it is not affected by the number $Q$ of decryption queries made by the adversary. This translates into instantiations where the adversary's advantage is bounded by a constant multiplied by the reduction's advantage in breaking the SD, DLIN or SXDH assumption. This stands in contrast with the security reduction of [57], which inherits the linear loss $O(Q)$ incurred by the dual system encryption paradigm [71,54]. However, we only consider tightness in the single-challenge setting. Our schemes are not proven tightly secure when the adversary obtains polynomially-many challenge ciphertexts as in $[9,42]$.

\section{Instantiations}

\subsection{A Construction in Groups of Composite Order $N=p_{1} p_{2}$}

The construction relies on a hash proof system in a group $\mathbb{G}$ of composite order $N=p_{1} p_{2}$ and it is conceptually close to the one in [43] (notably because it builds on a $\log p_{2}$-entropic hash proof system, as defined in [51]). The public key includes group elements $\left(g, X=g^{x}\right)$ in the subgroup $\mathbb{G}_{p_{1}}$ of order $p_{1}$ and the sets $\mathcal{C}$ and $\mathcal{V}$ are defined to be $\mathbb{G}$ and $\mathbb{G}_{p_{1}}$, respectively. The sampling algorithm returns $\Phi=g^{r} \in \mathbb{G}_{p_{1}}$ for a randomly chosen exponent $r \stackrel{R}{\leftarrow} \mathbb{Z}_{N}$, which allows publicly evaluating 
$H\left(X^{r}\right)=H\left(\Phi^{x}\right)$ using a pairwise independent hash function $H: \mathbb{G} \rightarrow\{0,1\}^{\ell}$. Since the public key is independent of $x \bmod p_{2}$, for any $\Phi \in \mathbb{G}$ that has a non-trivial component of order $p_{2}$, the "hash value" $\Phi^{x}$ has exactly $\log p_{2}$ bits of min-entropy and the leftover hash lemma implies that $H\left(\Phi^{x}\right)$ is statistically close to the uniform distribution in $\{0,1\}^{\ell}$ when $\ell$ is sufficiently small.

In order to turn the scheme into an all-but-one perfectly sound threshold HPS, we need a mechanism that proves membership in the subgroup $\mathbb{G}_{p_{1}}$ and guarantees the perfect soundness of proofs of membership for all tags tag $\in \mathbb{Z}_{N}$ such that tag $\neq$ tag $^{\star}$. To this end, we use additional public parameters $(u, v) \in \mathbb{G}^{2}$ and a tag-dependent group element $u^{\text {tag }} \cdot v$ will serve as a common reference string to generate a non-interactive proof that $\Phi \in \mathbb{G}_{p_{1}}$. Membership in $\mathbb{G}_{p_{1}}$ can be noninteractively proved using a technique that can be traced back to [37]. The proof consists of a group element $\pi_{\mathrm{SD}} \in \mathbb{G}$ satisfying the equality $e\left(\Phi, u^{\text {tag }} \cdot v\right)=e\left(g, \pi_{\mathrm{SD}}\right)$, which ensures that $\Phi \in \mathbb{G}_{p_{1}}$ as long as $u^{\text {tag }} \cdot v$ has a $\mathbb{G}_{p_{2}}$ component. In the public parameters produced by SetupABO, the value $u^{\text {tag }} \cdot v$ thus has to be in $\mathbb{G} \backslash \mathbb{G}_{p_{1}}$ for any tag $\neq \operatorname{tag}^{\star}$ in such a way that generating fake proofs that $\Phi \in \mathbb{G}_{p_{1}}$ is impossible. At the same time, $u^{\operatorname{tag}^{\star}} \cdot v$ should be in $\mathbb{G}_{p_{1}}$ so that fake proofs can be generated for tag*.

$\operatorname{SetupSound}(\lambda, t, n)$ : choose a group $\mathbb{G}$ of composite order $N=p_{1} p_{2}$ for large primes $p_{i}>2^{l(\lambda)}$ for each $i \in\{1,2\}$ and for some polynomial $l: \mathbb{N} \rightarrow \mathbb{N}$. Then, conduct the following steps

1. Pick $g \stackrel{R}{\leftarrow} \mathbb{G}_{p_{1}}, u, v \stackrel{R}{\leftarrow} \mathbb{G}, x \stackrel{R}{\leftarrow} \mathbb{Z}_{N}$ and set $X=g^{x} \in \mathbb{G}_{p_{1}}$.

2. Choose a random polynomial $P[Z] \in \mathbb{Z}_{N}[Z]$ of degree $t-1$ such that $P(0)=x$. For each $i \in\{1, \ldots, n\}$, compute $Y_{i}=g^{P(i)} \in \mathbb{G}_{p_{1}}$.

3. Select a pairwise independent hash function $H: \mathbb{G} \rightarrow\{0,1\}^{\ell}$, where $\ell \leq l(\lambda)-2 \lambda$. Note that the range $\mathcal{K}=\{0,1\}^{\ell}$ of $H$ forms a group for the bitwise exclusive OR operation $\odot=\oplus$.

4. Define private key shares $\left(\mathbf{s k}_{1}, \ldots, \mathrm{sk}_{n}\right)$ as $\mathbf{s k}_{i}=P(i) \in \mathbb{Z}_{N}$ for each $i=1$ to $n$. The vector $\left(\mathrm{vk}_{1}, \ldots, \mathrm{vk}_{n}\right)$ is defined as $\mathrm{vk}_{i}=Y_{i} \in \mathbb{G}_{p_{1}}$ for each $i$ and the public key consists of

$$
\mathrm{pk}=\left(\left(\mathbb{G}, \mathbb{G}_{T}\right), N, g, X, u, v, H\right) .
$$

In addition, we have $\left(\mathcal{C}, \mathcal{V}, \mathcal{K}, \mathcal{K}^{\prime}, \mathcal{R}\right)=\left(\mathbb{G}, \mathbb{G}_{p_{1}},\{0,1\}^{\ell}, \mathbb{G}, \mathbb{Z}_{N}\right)$.

$\operatorname{SetupABO}\left(\lambda, t, n, \operatorname{tag}^{\star}\right)$ : is identical to SetupSound with the difference that, instead of being chosen uniformly in $\mathbb{G}, v$ is defined as $v=u^{-\operatorname{tag}^{\star}} \cdot g^{\alpha}$ for some random $\alpha \stackrel{R}{\leftarrow} \mathbb{Z}_{N}$. The algorithm also outputs the simulation trapdoor $\tau=\alpha \in \mathbb{Z}_{N}$.

Sample(pk): parse pk as $\left(\left(\mathbb{G}, \mathbb{G}_{T}\right), N, g, X, u, v, H\right)$. Choose $r \stackrel{R}{\leftarrow} \mathbb{Z}_{N}$, compute $\Phi=g^{r} \in \mathbb{G}_{p_{1}}$ and output the pair $(r, \Phi) \in \mathbb{Z}_{N} \times \mathbb{G}_{p_{1}}$.

Prove $(\mathrm{pk}, \operatorname{tag}, r, \Phi)$ : parse pk as $\left(\left(\mathbb{G}, \mathbb{G}_{T}\right), N, g, X, u, v, H\right)$ and return $\perp$ if $\Phi \neq g^{r}$. Otherwise, compute and return $\pi_{\mathrm{SD}}=\left(u^{\mathrm{tag}} \cdot v\right)^{r}$.

$\operatorname{SimProve}(\mathrm{pk}, \tau, \operatorname{tag}, \Phi)$ : return $\perp$ if $\operatorname{tag} \neq \operatorname{tag}^{\star}$ or if $\Phi \notin \mathbb{G}$. Otherwise, use the simulation trapdoor $\tau=\alpha \in \mathbb{Z}_{N}$ to compute and output $\pi_{\mathrm{SD}}=\Phi^{\alpha}$.

Verify $\left(\mathrm{pk}, \operatorname{tag}, \Phi, \pi_{\mathrm{SD}}\right)$ : return 1 if and only if $\left(\Phi, \pi_{\mathrm{SD}}\right) \in \mathbb{G}^{2}$ and $e\left(\Phi, u^{\mathrm{tag}} \cdot v\right)=e\left(g, \pi_{\mathrm{SD}}\right)$.

PubEval (pk, $r, \Phi)$ : on input of pk $=\left(\left(\mathbb{G}, \mathbb{G}_{T}\right), N, g, X, u, v, H\right)$, return $\perp$ if $(r, \Phi) \notin \mathbb{Z}_{N} \times \mathbb{G}$. Otherwise, compute and return $K=H\left(X^{r}\right) \in\{0,1\}^{\ell}$.

SharePrivEval $\left(\mathrm{pk}^{\mathrm{sk}}, \Phi\right)$ : return $\perp$ if $\Phi \notin \mathbb{G}$. Otherwise, compute and return $\left(K_{i}, \pi_{K_{i}}\right)$, where $K_{i}=\Phi^{\text {sk }}{ }_{i}=\Phi^{P(i)}$ and $\pi_{K_{i}}=\varepsilon$ is simply the empty string.

ShareEvalVerify $\left(\mathrm{pk}, \mathrm{vk}_{i}, \Phi, K_{i}, \pi_{K_{i}}\right)$ : if $K_{i} \notin \mathbb{G}, \mathrm{vk}_{i} \notin \mathbb{G}$ or $\pi_{K_{i}} \neq \varepsilon$, return 0. Otherwise, return 1 if $e\left(g, K_{i}\right)=e\left(\Phi, \mathbf{v k}_{i}\right)$. In any other situation, return 0 (the proof $\pi_{K_{i}}$ is completely ignored in this instantiation since, given $\mathrm{vk}_{i}=Y_{i}$, the private evaluation share $K_{i}$ is directly verifiable). 
Combine $\left(\mathrm{pk}, \Phi,\left\{\left(K_{i}, \pi_{K_{i}}\right)\right\}_{i \in S}\right)$ : return $\perp$ if ShareEvalVerify $\left(\mathrm{pk}, \mathrm{vk}_{i}, \Phi, K_{i}, \pi_{K_{i}}\right)=0$ for some $i \in S$. Otherwise, compute $K=H\left(\prod_{i \in S} K_{i}^{\Delta_{i, S}(0)}\right)=H\left(\Phi^{x}\right) \in \mathcal{K}$ by interpolation in the exponent.

In the proof of Theorem 2, we rely on the Leftover Hash Lemma [41] to establish the universality property of the above construction.

Lemma 1 ([41]). Let $X \in \mathcal{X}$ be a random variable such that $H_{\infty}(X) \geq k$ and let $\mathcal{H}$ be a family of pairwise independent hash functions with domain $\mathcal{X}$ and range $\{0,1\}^{\ell}$. Then, if $H \stackrel{R}{\leftarrow} \mathcal{H}$, we have

$$
\Delta\left((H, H(X)),\left(H, U_{\ell}\right)\right) \leq 1 / 2^{(k-\ell) / 2},
$$

where $U_{\ell}$ denotes the uniform distribution over $\{0,1\}^{\ell}$.

Theorem 2. The above construction is an all-but-one perfectly sound threshold hash proof system if the $S D$ assumption holds in $\mathbb{G}$.

Proof. The theorem is proved by demonstrating that, under the Subgroup Decision assumption, the scheme provides all the properties required from an all-but-one perfectly sound HPS.

The subset membership hardness property is straightforward as it is exactly the Subgroup Decision assumption in this instantiation. The simulatability of share proofs is also trivial to verify since no non-interactive proof is needed to check the validity of private evaluation shares. We thus focus on remaining properties.

SETUP Indistinguishability. The only difference between the outputs ( $\mathbf{p k},\left\{\mathbf{s k}_{i}\right\}_{i=1}^{n},\left\{\mathrm{vk}_{i}\right\}_{i=1}^{n}$ ) of SetupSound $(\lambda, t, n)$ and $\operatorname{SetupABO}\left(\lambda, t, n, \operatorname{tag}^{\star}\right)$ is the distribution of $v \in \mathbb{G}$ which is uniform in $\mathbb{G}$ in the former case and equals $v=u^{-\operatorname{tag}^{\star}} \cdot g^{\alpha}$, where $\alpha \stackrel{R}{\leftarrow} \mathbb{Z}_{N}$, when it is returned by SetupABO. In the latter situation, $v$ can be seen a Boneh-Goh-Nissim encryption [16] of - tag $^{\star}$ whereas a uniformly random $v \in_{R} \mathbb{G}$ can be interpreted as a BGN encryption of a random plaintext. Consequently, the public outputs of SetupSound and SetupABO cannot be told apart if the Subgroup Decision assumption (which is equivalent to the semantic security of the BGN cryptosystem) holds. More formally, we have the following result:

Claim 1. Under the SD assumption, no PPT adversary can distinguish the output of SetupSound from SetupABO. For any PPT setup distinguisher $\mathcal{D}$, we can build an SD distinguisher $\mathcal{B}$ with comparable running time such that $\mathbf{A d v}_{\mathcal{D}}^{\text {Setup-indist }}(\lambda) \leq \mathbf{A d v}_{\mathcal{B}}^{\mathrm{SD}}(\lambda)$.

Proof. The proof is completely similar to [16][Theorem 3.1]. Let us assume that an adversary can distinguish the public outputs of SetupSound and SetupABO. We build a distinguisher $\mathcal{B}$ that takes as input $\left(g \in \mathbb{G}_{p_{1}}, h \in \mathbb{G}\right)$ and $\eta$ with the goal of deciding if $\eta \in_{R} \mathbb{G}$ or $\eta \in_{R} \mathbb{G}_{p_{1}}$. To this end, $\mathcal{B}$ defines pk $=\left(\left(\mathbb{G}, \mathbb{G}_{T}\right), N, g, X, u, v, H\right)$ where $X=g^{x}$, for some $x \stackrel{R}{\leftarrow} \mathbb{Z}_{N}, u \stackrel{R}{\leftarrow} \mathbb{G}$ and $v=u^{- \text {tag }} \cdot \eta$. If $\eta \in_{R} \mathbb{G}$, the distribution of pk is identical to that of an output of SetupSound. If $\eta \in_{R} \mathbb{G}_{p_{1}}, v$ is distributed as a public output of $\operatorname{SetupABO}\left(\lambda, t, n, \operatorname{tag}^{\star}\right)$ since we can write $v=u^{-\operatorname{tag}^{\star}} \cdot g^{\alpha}$, for some uniformly random $\alpha \in_{R} \mathbb{Z}_{p_{1}}$.

Correctness and Public Evaluability on $\mathcal{V}$. Since the public values pk $=g^{x}$ and $\mathrm{vk}_{i}=g^{P(i)}$ uniquely determine $P[X] \bmod p_{1}$ as well as $\mathbf{s k}_{i} \bmod p_{1}$, for any $\Phi \in \mathbb{G}_{p_{1}}$, there is only one possible 
value SharePrivEval $\left(\mathrm{pk}^{\mathrm{sk}}, \Phi_{i}\right)=\left(K_{i}, \varepsilon\right)=\left(\Phi^{P(i)}, \varepsilon\right)$. The second condition is immediate to verify.

Universality. Let $\Phi$ be a random element of order $N$ in $\mathbb{G}$. For any $(t-1)$-subset $\bar{S} \subset\{1, \ldots, n\}$, if we consider the min-entropy of $\Phi^{x}$ given $\Phi, g^{x},\left\{\mathbf{v k}_{i}=g^{P(i)}\right\}_{i=1}^{n}$ and $\left\{\mathbf{s k}_{i}=P(i)\right\}_{i \in \bar{S}}$, we have

$$
\begin{aligned}
H_{\infty}\left(\Phi^{x} \mid\left(g, \Phi, g^{x},\left\{\mathbf{v k}_{i}\right\}_{i=1}^{n},\left\{\mathbf{s k}_{i}\right\}_{i \in \bar{S}}\right)\right) & =H_{\infty}\left(\Phi^{x} \mid\left(g, \Phi, g^{x}\right)\right)=H_{\infty}\left(x \bmod N \mid x \bmod p_{1}\right) \\
& =H_{\infty}\left(x \bmod p_{2} \mid x \bmod p_{1}\right)=H_{\infty}\left(x \bmod p_{2}\right)=\log p_{2} .
\end{aligned}
$$

Lemma 1 tells us that the statistical distance

$$
\Delta\left[\left(\left(g^{x},\left\{\mathrm{vk}_{i}\right\}_{i=1}^{n},\left\{s k_{i}\right\}_{i \in \bar{S}}, \Phi\right), H, H\left(\Phi^{x}\right)\right),\left(\left(g^{x},\left\{\mathrm{vk}_{i}\right\}_{i=1}^{n},\left\{s k_{i}\right\}_{i \in \bar{S}}, \Phi\right), H, U_{\ell}\right)\right],
$$

is smaller than $1 / 2^{\left(\log p_{2}-\ell\right) / 2}<1 / 2^{(l(\lambda)-\ell) / 2}$, which is negligible as long as $l(\lambda) \geq 2 \lambda+\ell$.

All-But-One Soundness. Since SetupABO chooses $u$ at random in $\mathbb{G}, u$ has a non-trivial $\mathbb{G}_{p_{2}}$ component with overwhelming probability. We know that $u^{\operatorname{tag}} \cdot v=u^{\text {tag-tag }}{ }^{\star} \cdot g^{\alpha}$ has a non-trivial $\mathbb{G}_{p_{2}}$ component whenever tag $\neq \operatorname{tag}^{\star}$. The equality $e\left(\Phi, u^{\operatorname{tag}} \cdot v\right)=e\left(g, \pi_{\mathrm{SD}}\right)$ - more precisely, the fact that its right-hand-side member has order $p_{1}$ - thus guarantees that $\Phi \in \mathbb{G}_{p_{1}}$ as long as $\operatorname{tag} \neq \operatorname{tag}^{\star}$. At the same time, $u^{\operatorname{tag} g^{\star}} \cdot v=g^{\alpha}$ has order $p_{1}$, and the trapdoor allows simulating proofs that $\Phi \in \mathbb{G}_{p_{1}}$. When $\Phi$ is really in the subgroup $\mathbb{G}_{p_{1}}, \pi_{\mathrm{SD}}=\Phi^{\alpha}$ equals the proof that would be produced using the real witness $r=\log _{g}(\Phi)$. When, $\Phi \in_{R} \mathbb{G}, \pi_{\mathrm{SD}}=\Phi^{\alpha}$ still satisfies the equality $e\left(\Phi, u^{\operatorname{tag}^{\star}} \cdot v\right)=e\left(g, \pi_{\mathrm{SD}}\right)$ and can thus serve as a simulated proof that $\Phi \in \mathbb{G}_{p_{1}}$.

Consistency. Let us assume that a PPT adversary $\mathcal{A}$ can break the consistency property of the all-but-one HPS with non-negligible probability. We show that $\mathcal{A}$ implies a distinguisher $\mathcal{B}$ for the Subgroup Decision assumption. The distinguisher $\mathcal{B}$ receives $\left(g \in \mathbb{G}_{p_{1}}, h \in \mathbb{G}\right)$ and $\eta \in \mathbb{G}$ with the aim of deciding if $\eta$ has a non-trivial $\mathbb{G}_{p_{2}}$ component. This is done by generating the public key pk using $g \in \mathbb{G}_{p_{1}}$ and $h \in \mathbb{G}$ and by choosing $\left\{\left(\mathbf{s k}_{i}, \mathrm{vk}_{i}\right)\right\}_{i=1}^{n}$ as in the specification of the scheme.

The only way for the adversary to break the consistency property is to output $\left(\operatorname{tag}, \Phi, \pi_{\mathrm{SD}}\right)$ (note that $\Phi$ 's membership in $\mathbb{G}_{p_{1}}$ is guaranteed by the perfectly sound proof $\pi_{\mathrm{SD}}$ ) and two sets of decryption shares where at least one share is of the form $K_{i}=\Phi^{P(i)} \cdot R_{2, i}$, for some $R_{2, i} \in \mathbb{G}_{p_{2}}^{*}$. Since $\mathcal{B}$ knows sk ${ }_{i}=P(i)$, it can compute $R_{2, i}=K_{i} / \Phi^{P(i)} \in \mathbb{G}_{p_{2}}$, which allows deciding whether $\eta \in \mathbb{G}_{p_{1}}$ by testing whether the equality $e\left(\eta, R_{2, i}\right)=1_{\mathbb{G}_{T}}$ (which only holds if $\eta \in \mathbb{G}_{p_{1}}$ ) holds.

Subset Membership Hardness. We prove that, under the SD assumption, no PPT adversary can distinguish the distributions

$$
D_{0}:=\left\{\left(\mathrm{pk},\left\{\left(\mathrm{sk}_{i}, \mathrm{vk}_{i}\right)\right\}_{i=1}^{n}, \mathcal{C}, \mathcal{V}, C, \tau\right) \mid C \stackrel{R}{\leftarrow} \mathcal{V}\right\}, \quad D_{1}:=\left\{\left(\mathrm{pk},\left\{\left(\mathrm{sk}_{i}, \mathrm{vk}_{i}\right)\right\}_{i=1}^{n}, \mathcal{C}, \mathcal{V}, C, \tau\right) \mid C \stackrel{R}{\leftarrow} \mathcal{C} \backslash \mathcal{V}\right\}
$$

Namely, we prove that any subset membership adversary $\mathcal{A}$ implies a distinguisher $\mathcal{B}^{\mathrm{SM}}$ for the SD assumption such that $\mathbf{A d v}_{\mathcal{A}}^{\mathrm{SM}}(\lambda) \leq \mathbf{A} \mathbf{d v}_{\mathcal{B}^{\mathrm{SM}}}^{\mathrm{SD}}(\lambda)$.

The reduction $\mathcal{B}^{\mathrm{SM}}$ inputs $\left(g \in \mathbb{G}_{p_{1}}, h \in \mathbb{G}, \eta\right)$ and undertakes to decide if $\eta \in_{R} \mathbb{G}$ or $\eta \in_{R} \mathbb{G}_{p_{1}}$. It chooses a pairwise independent hash function $H: \mathbb{G} \rightarrow\{0,1\}^{\ell}$. It also samples $x \stackrel{R}{\leftarrow} \mathbb{Z}_{N}$ and picks a random polynomial $P[Z]$ of degree $t-1$ such that $P(0)=x$ and uses these to define

$$
\mathrm{pk}=\left(\left(\mathbb{G}, \mathbb{G}_{T}\right), N, g, X=g^{x}, u, v, H\right), \quad\left\{\left(\mathrm{sk}_{i}, \mathrm{vk}_{i}\right)=\left(P(i), g^{P(i)}\right)\right\}_{i=1}^{n}
$$


where $u=h$ and $v=u^{-\operatorname{tag}^{\star}} \cdot g^{\alpha}$, for a randomly chosen $\alpha \stackrel{R}{\leftarrow} \mathbb{Z}_{N}$. Note that the distribution of pk and $\left\{\left(\mathbf{s k}_{i}, \mathrm{vk}_{i}\right)\right\}_{i=1}^{n}$ matches exactly the output distribution of $\operatorname{SetupABO}\left(\lambda, t, n, \operatorname{tag}^{\star}\right)$. Finally, $\mathcal{B}^{\mathrm{SM}}$ sets $C=\eta, \tau=\alpha$ and outputs the tuple $D:=\left(\right.$ pk, $\left.\left\{\left(\mathrm{sk}_{i}, \mathrm{vk}_{i}\right)\right\}_{i=1}^{n}, \mathcal{C}, \mathcal{V}, C, \tau\right)$. Clearly, if $\eta \in_{R} \mathbb{G}_{p_{1}}$, $D$ is a random sample from the distribution $D_{0}$. If $\eta \in_{R} \mathbb{G}$, with overwhelming probability, it has a non-trivial $\mathbb{G}_{p_{2}}$ component, so that $D$ matches the distribution $D_{1}$.

When the above all-but-one perfectly sound threshold hash proof system is plugged into the generic construction of Section 4, the resulting threshold cryptosystem bears resemblance with the scheme in [57], which makes use of groups whose order is a product of three primes. However, it is more efficient and its security proof is completely different as the dual system encryption approach [71] is not used here.

\subsection{A Construction from the Decision Linear Assumption in Prime Order Groups}

This section presents an all-but-one threshold hash proof system based on the DLIN assumption in prime order bilinear groups. The public key comprises elements $\left(g, g_{1}, g_{2}, X_{1}, X_{2}\right) \in \mathbb{G}^{5}$, where $X_{1}=g_{1}^{x_{1}} \cdot g^{z}, X_{2}=g_{2}^{x_{2}} \cdot g^{z}$ and $\left(x_{1}, x_{2}, z\right)$ are part of the private key. The sets $\mathcal{C}$ and $\mathcal{V} \subset \mathcal{C}$ consist of $\mathcal{C}=\mathbb{G}^{3}$ and $\mathcal{V}=\left\{\left(\Phi_{1}, \Phi_{2}, \Phi_{3}\right)=\left(g_{1}^{\theta_{1}}, g_{2}^{\theta_{2}}, g^{\theta_{1}+\theta_{2}}\right) \mid \theta_{1}, \theta_{2} \in \mathbb{Z}_{p}\right\}$, respectively. For any $\Phi=\left(\Phi_{1}, \Phi_{2}, \Phi_{3}\right) \in \mathcal{V}$, the public evaluation algorithm computes $X_{1}^{\theta_{1}} \cdot X_{2}^{\theta_{2}}$, which can be privately evaluated as $\Phi_{1}^{x_{1}} \cdot \Phi_{2}^{x_{2}} \cdot \Phi_{3}^{z}$.

As in the previous instantiation, we append to elements $\Phi \in \mathcal{V}$ a non-interactive proof of their membership of $\mathcal{V}$ (i.e., a proof that $\left(g, g_{1}, g_{2}, \Phi_{1}, \Phi_{2}, \Phi_{3}\right)$ is a linear tuple) and, in this case, the proof is obtained using the Groth-Sahai techniques. However, we cannot simply combine them with a DLIN-based hash proof system in the obvious way. The reason is that, using parameters produced by SetupABO and under the special tag $\operatorname{tag}^{\star}$, SimProve must be able to compute a fake noninteractive proof of the statement $\Phi \in \mathcal{V}$ for an element $\Phi \notin \mathcal{V}$. At the same time, we should make sure that, for any tag such that $\operatorname{tag} \neq \operatorname{tag}^{\star}$, it will be impossible to simulate such proofs. To solve this problem, we need a form of one-time simulation soundness [65] which can be possibly obtained from Groth's simulation-sound non-interactive proofs [38] or a more efficient variant suggested by Katz and Vaikuntanathan [49]. However, the specific language that we consider allows for even more efficient constructions: it is actually possible to build on the Groth-Sahai proofs, essentially without any loss of efficiency.

The solution is as follows. After having sampled a linear tuple $\Phi=\left(\Phi_{1}, \Phi_{2}, \Phi_{3}\right) \in \mathcal{V}$, the sampler generates his proof using a Groth-Sahai CRS that depends on tag. Algorithm SetupABO produces parameters in the fashion of the all-but-one technique [12]: the tag-based CRS is perfectly WI on the special tag tag* (which allows generating NIZK proofs for this tag) and perfectly sound for any other tag, which makes it impossible to convincingly prove false statements on tags tag $\neq \operatorname{tag}^{\star}$. Malkin, Teranishi, Vahlis and Yung [60] used a similar idea of message-dependent CRS in the context of signatures. A difference with [60] is that we do not need to extract witnesses from adversariallygenerated proofs and only use them as proofs of membership.

Interestingly, the same technique can be applied to have a more efficient simulation-sound proof of plaintext equality in the Naor-Yung-type [61] cryptosystem in [49][Section 3.2.2]: the proof can be reduced from 60 to 22 group elements and the ciphertext size is decreased by more than $50 \%$.

$\operatorname{SetupSound}(\lambda, t, n)$ : Choose a group $\mathbb{G}$ of prime order $p>2^{\lambda}$ with generators $g, g_{1}, g_{2}, f_{1}, f_{2} \stackrel{R}{\leftarrow} \mathbb{G}$. 
1. Choose $x_{1}, x_{2}, z \stackrel{R}{\leftarrow} \mathbb{Z}_{p}$ and set $X_{1}=g_{1}^{x_{1}} g^{z}, X_{2}=g_{2}^{x_{2}} g^{z}$. Define the vectors $\vec{g}_{1}=\left(g_{1}, 1, g\right)$ and $\vec{g}_{2}=\left(1, g_{2}, g\right)$. Then, pick $\xi_{1}, \xi_{2} \stackrel{R}{\leftarrow} \mathbb{Z}_{p}$ and define $\vec{g}_{3}=\vec{g}_{1} \xi_{1} \cdot \vec{g}_{2} \xi_{2}$.

2. Choose $\phi_{1}, \phi_{2} \stackrel{R}{\leftarrow} \mathbb{Z}_{p}$ and define $\overrightarrow{f_{1}}=\left(f_{1}, 1, g\right), \vec{f}_{2}=\left(1, f_{2}, g\right)$ and $\vec{f}_{3}=\vec{f}_{1}^{\phi_{1}} \cdot \vec{f}_{2}^{\phi_{2}} \cdot(1,1, g)$.

3. Choose random polynomials $P_{1}[Z], P_{2}[Z], P[Z] \in \mathbb{Z}_{p}[Z]$ of degree $t-1$ such that $P_{1}(0)=x_{1}$, $P_{2}(0)=x_{2}$ and $P(0)=z$. For each $i=1$ to $n$, compute $Y_{i, 1}=g_{1}^{P_{1}(i)} g^{P(i)}, Y_{i, 2}=g_{2}^{P_{2}(i)} g^{P(i)}$.

4. Define private key shares $\mathbf{S K}=\left(\mathbf{s k}_{1}, \ldots, \mathbf{s k}_{n}\right)$ as $\mathbf{s k}_{i}=\left(P_{1}(i), P_{2}(i), P(i)\right) \in\left(\mathbb{Z}_{p}\right)^{3}$ for each $i \in\{1, \ldots, n\}$. Verification keys $\mathbf{V K}=\left(\mathbf{v k}_{1}, \ldots, \mathbf{v k}_{n}\right)$ are defined as $\mathbf{v k}_{i}=\left(Y_{i, 1}, Y_{i, 2}\right) \in \mathbb{G}^{2}$ for each $i \in\{1, \ldots, n\}$ and the public key is defined to be

$$
\mathrm{pk}=\left(\left(\mathbb{G}, \mathbb{G}_{T}\right), g, \overrightarrow{g_{1}}, \overrightarrow{g_{2}}, \overrightarrow{g_{3}}, \overrightarrow{f_{1}}, \overrightarrow{f_{2}}, \overrightarrow{f_{3}}, X_{1}, X_{2}\right) .
$$

As for the sets $\left(\mathcal{C}, \mathcal{K}, \mathcal{K}^{\prime}, \mathcal{R}\right)$, they are defined as $\mathcal{C}=\mathbb{G}^{3}, \mathcal{K}=\mathcal{K}^{\prime}=\mathbb{G}$ and $\mathcal{R}=\left(\mathbb{Z}_{p}\right)^{2}$, respectively. The subset $\mathcal{V} \subset \mathcal{C}$ consists of the language $\left(\Phi_{1}, \Phi_{2}, \Phi_{3}\right) \in \mathbb{G}^{3}$ for which there exists $\theta_{1}, \theta_{2} \in \mathbb{Z}_{p}$ such that $\Phi_{1}=g_{1}^{\theta_{1}}, \Phi_{2}=g_{2}^{\theta_{2}}$ and $\Phi_{3}=g^{\theta_{1}+\theta_{2}}$.

$\operatorname{SetupABO}\left(\lambda, t, n, \operatorname{tag}^{\star}\right)$ : is identical to SetupSound with the following differences.

1. In step $1, \overrightarrow{g_{3}}$ is set as $\overrightarrow{g_{3}}=\overrightarrow{g_{1}} \xi_{1} \cdot \overrightarrow{g_{2}} \xi_{2} \cdot(1,1, g)^{- \text {tag }}$ instead of being chosen in $\operatorname{span}\left(\overrightarrow{g_{1}}, \overrightarrow{g_{2}}\right)$.

2 . In step 2 , the vectors $\left(\overrightarrow{f_{1}}, \overrightarrow{f_{2}}, \overrightarrow{f_{3}}\right)$ are chosen so as to have $\vec{f}_{3}=\vec{f}_{1}^{\phi_{1}} \cdot \vec{f}_{2}{ }^{\phi_{2}}$.

3. The algorithm additionally outputs the trapdoor $\tau=\left(\xi_{1}, \xi_{2}, \phi_{1}, \phi_{2}\right) \in\left(\mathbb{Z}_{p}\right)^{4}$.

Sample(pk): choose $\theta_{1}, \theta_{2} \stackrel{R}{\leftarrow} \mathbb{Z}_{p}$, compute $\Phi=\left(\Phi_{1}, \Phi_{2}, \Phi_{3}\right)=\left(g_{1}^{\theta_{1}}, g_{2}^{\theta_{2}}, g^{\theta_{1}+\theta_{2}}\right)$ and output $\left(\left(\theta_{1}, \theta_{2}\right), \Phi\right)$.

Prove (pk, tag, $\left.\left(\theta_{1}, \theta_{2}\right), \Phi\right)$ : parse pk as $\left(\left(\mathbb{G}, \mathbb{G}_{T}\right), g, \overrightarrow{g_{1}}, \overrightarrow{g_{2}}, \overrightarrow{g_{3}}, \overrightarrow{f_{1}}, \overrightarrow{f_{2}}, \overrightarrow{f_{3}}, X_{1}, X_{2}\right)$ and $\Phi$ as $\left(\Phi_{1}, \Phi_{2}, \Phi_{3}\right)$. Construct ${ }^{1}$ a vector $\vec{g}_{\mathrm{tag}}=\overrightarrow{g_{3}} \cdot(1,1, g)^{\mathrm{tag}}$ and use $\mathbf{g}_{\mathrm{tag}}=\left(\overrightarrow{g_{1}}, \overrightarrow{g_{2}}, \vec{g}_{\mathrm{tag}}\right)$ as a Groth-Sahai CRS to generate a NIZK proof that $\left(g, g_{1}, g_{2}, \Phi_{1}, \Phi_{2}, \Phi_{3}\right)$ is a linear tuple. More precisely, generate commitments $\vec{C}_{\theta_{1}}, \vec{C}_{\theta_{2}}$ to exponents $\theta_{1}, \theta_{2} \in \mathbb{Z}_{p}$ (in other words, compute $\vec{C}_{\theta_{i}}=\vec{g}_{\text {tag }} \theta_{i} \cdot \overrightarrow{g_{1}} \vec{r}_{i} \cdot \overrightarrow{g_{2}} s_{i}$ with $r_{i}, s_{i} \stackrel{R}{\leftarrow} \mathbb{Z}_{p}$ for each $\left.i \in\{1,2\}\right)$ and a proof $\pi_{\left(\theta_{1}, \theta_{2}\right)}$ that they satisfy

$$
\Phi_{1}=g_{1}^{\theta_{1}}, \quad \Phi_{2}=g_{2}^{\theta_{2}}, \quad \Phi_{3}=g^{\theta_{1}+\theta_{2}} .
$$

The whole proof $\pi_{\text {LIN }}$ for (3) consists of $\vec{C}_{\theta_{1}}, \vec{C}_{\theta_{2}}$ and $\pi_{\left(\theta_{1}, \theta_{2}\right)}$ (see Appendix B.1 for details about the generation of this proof) and requires 12 elements of $\mathbb{G}$.

$\operatorname{SimProve}(\mathrm{pk}, \tau, \operatorname{tag}, \Phi)$ : parses pk as above, $\tau$ as $\left(\xi_{1}, \xi_{2}, \phi_{1}, \phi_{2}\right) \in\left(\mathbb{Z}_{p}\right)^{4}$ and $\Phi$ as $\left(\Phi_{1}, \Phi_{2}, \Phi_{3}\right) \in \mathbb{G}^{3}$. If tag $\neq \operatorname{tag}^{\star}$, return $\perp$. Otherwise, the commitments $\vec{C}_{\theta_{1}}, \vec{C}_{\theta_{2}}$ and the proof $\pi_{\text {LIN }}$ must be generated for the CRS $\mathbf{g}_{\text {tag }^{\star}}=\left(\overrightarrow{g_{1}}, \overrightarrow{g_{2}}, \vec{g}_{\mathrm{tag}^{\star}}\right)$, where $\vec{g}_{\mathrm{tag}^{\star}}=\overrightarrow{g_{3}} \cdot(1,1, g)^{\operatorname{tag}^{\star}}=\overrightarrow{g_{1}} \vec{\xi}_{1} \cdot{\overrightarrow{g_{2}}}^{\xi_{2}}$, which is a Groth-Sahai CRS for the witness indistinguishability setting.

1. Using the trapdoor $\left(\xi_{1}, \xi_{2}\right)$, simulate proofs for multi-exponentiation equations (see Appendix B.1 for details as to how such proofs can be simulated). That is, generate $\vec{C}_{\theta_{1}}, \vec{C}_{\theta_{2}}$ as commitments to 0 and compute $\pi_{\left(\theta_{1}, \theta_{2}\right)}$ as a simulated proof that relations (3) hold.

2. Output $\pi_{\mathrm{LIN}}=\left(\vec{C}_{\theta_{1}}, \vec{C}_{\theta_{2}}, \pi_{\left(\theta_{1}, \theta_{2}\right)}\right)$ that consists of perfectly hiding commitments and simulated NIZK proofs which, on the CRS $\left(\overrightarrow{g_{1}}, \overrightarrow{g_{2}}, \vec{g}_{\mathrm{tag}}\right)$, are distributed as real proofs.

$\operatorname{Verify}\left(\mathrm{pk}, \operatorname{tag}, \Phi, \pi_{\mathrm{LIN}}\right)$ : parse pk and $\Phi$ as above and $\pi_{\mathrm{LIN}}$ as $\left(\vec{C}_{\theta_{1}}, \vec{C}_{\theta_{2}}, \pi_{\left(\theta_{1}, \theta_{2}\right)}\right) \in \mathbb{G}^{12}$. Then, compute $\vec{g}_{\text {tag }}=\overrightarrow{g_{3}} \cdot(1,1, g)^{\text {tag }}$ and use $\mathbf{g}_{\text {tag }}=\left(\overrightarrow{g_{1}}, \overrightarrow{g_{2}}, \vec{g}_{\mathrm{tag}}\right)$ as a Groth-Sahai CRS to verify the proof $\pi_{\text {LIN }}$. If the latter is deemed as a valid proof for the relations (3), return 1 . Otherwise, return 0.

\footnotetext{
${ }^{1}$ We assume that tags are non-zero. This can be enforced by having Prove and Verify output $\perp$ when tag $=0$.
} 
$\operatorname{PubEval}\left(\mathrm{pk},\left(\theta_{1}, \theta_{2}\right), \Phi\right)$ : parse pk and $\Phi$ as above. Return $\perp$ if $\left(\Phi_{1}, \Phi_{2}, \Phi_{3}\right) \neq\left(g_{1}^{\theta_{1}}, g_{2}^{\theta_{2}}, g^{\theta_{1}+\theta_{2}}\right)$. Otherwise, compute and return $K=X_{1}^{\theta_{1}} \cdot X_{2}^{\theta_{2}} \in \mathcal{K}$.

SharePrivEval $\left(\mathrm{pk} \mathrm{sk}_{i}, \Phi\right)$ : parse $\mathrm{sk}_{i}$ as $\left(P_{1}(i), P_{2}(i), P(i)\right) \in\left(\mathbb{Z}_{p}\right)^{3}$ and return $\perp$ if $\Phi \notin \mathbb{G}^{3}$. Otherwise, compute and return a pair $\left(K_{i}, \pi_{K_{i}}\right)$, where $K_{i}=\Phi_{1}^{P_{1}(i)} \cdot \Phi_{2}^{P_{2}(i)} \cdot \Phi_{3}^{P(i)} \in \mathcal{K}^{\prime}$ and $\pi_{K_{i}}=\left(\vec{C}_{P_{1}}, \vec{C}_{P_{2}}, \vec{C}_{P}, \pi_{K_{i}}^{\prime}\right) \in \mathbb{G}^{15}$ is a proof consisting of commitments $\vec{C}_{P_{1}}, \vec{C}_{P_{2}}, \vec{C}_{P}$ to exponents $P_{1}(i), P_{2}(i), P(i) \in \mathbb{Z}_{p}$ and a proof $\pi_{K_{i}}^{\prime}$ that these satisfy the equations

$$
K_{i}=\Phi_{1}^{P_{1}(i)} \cdot \Phi_{2}^{P_{2}(i)} \cdot \Phi_{3}^{P(i)}, \quad Y_{i, 1}=g_{1}^{P_{1}(i)} g^{P(i)}, \quad Y_{i, 2}=g_{2}^{P_{2}(i)} g^{P(i)} .
$$

The perfectly binding commitments $\vec{C}_{P_{1}}, \vec{C}_{P_{2}}, \vec{C}_{P}$ and the proof $\pi_{K_{i}}^{\prime}$ are generated using the vectors $\mathbf{f}=\left(\overrightarrow{f_{1}}, \overrightarrow{f_{2}}, \overrightarrow{f_{3}}\right)$ as a Groth-Sahai CRS (in such a way that $\vec{C}_{P_{1}}=\vec{f}_{3}{ }^{P_{1}(i)} \cdot \vec{f}_{1}^{r_{P_{1}}} \cdot \vec{f}_{2}{ }^{s_{P_{1}}}$, for some $r_{P_{1}}, s_{P_{1}} \stackrel{R}{\leftarrow} \mathbb{Z}_{p}$, for example).

ShareEvalVerify $\left(\mathrm{pk}, \mathrm{vk}_{i}, \Phi, K_{i}, \pi_{K_{i}}\right):$ parse $\mathrm{vk}_{i}$ as $\left(Y_{i, 1}, Y_{i, 2}\right) \in \mathbb{G}^{2}$ and return $\perp$ if $\left(K_{i}, \pi_{K_{i}}\right)$ cannot be parsed as a tuple in $\mathbb{G} \times \mathbb{G}^{15}$. Otherwise, parse $\pi_{K_{i}}$ as $\pi_{K_{i}}=\left(\vec{C}_{P_{1}}, \vec{C}_{P_{2}}, \vec{C}_{P}, \pi_{K_{i}}^{\prime}\right) \in \mathbb{G}^{15}$ and return 1 if $\pi_{K_{i}}^{\prime}$ is a valid proof for equations (4). In any other situation, return 0 .

Combine(pk, $\left.\Phi,\left\{\left(K_{i}, \pi_{K_{i}}\right)\right\}_{i \in S}\right)$ : return $\perp$ if ShareEvalVerify $\left(\mathrm{pk}, \mathrm{vk}_{i}, \Phi, K_{i}, \pi_{K_{i}}\right)=0$ for some $i \in S$. Otherwise, compute $K=\prod_{i \in S} K_{i}^{\Delta_{i, S}(0)}=\Phi_{1}^{x_{1}} \cdot \Phi_{2}^{x_{2}} \cdot \Phi_{3}^{z} \in \mathcal{K}$.

Theorem 3. The above construction is an all-but-one perfectly sound threshold hash proof system assuming that the DLIN assumption holds in $\mathbb{G}$.

Proof. We show that, under the Decision Linear assumption, the scheme meets all the requirements of all-but-one perfectly sound threshold HPS. The subset membership hardness property is trivially implied by the DLIN assumption and we thus focus on remaining properties.

Setup Indistinguishability. The difference between the public outputs of SetupSound $(\lambda, t, n)$ and $\operatorname{SetupABO}\left(\lambda, t, n, \operatorname{tag}^{\star}\right)$ is in the distributions of vectors $\overrightarrow{g_{3}}$ and $\overrightarrow{f_{3}}$ since SetupSound chooses $\overrightarrow{g_{3}}$ in $\operatorname{span}\left(\overrightarrow{g_{1}}, \overrightarrow{g_{2}}\right)$ and $\overrightarrow{f_{3}} \notin \operatorname{span}\left(\overrightarrow{f_{1}}, \overrightarrow{f_{2}}\right)$ whereas SetupABO proceeds the other way around. For any PPT distinguisher $\mathcal{D}$ between the outputs of SetupSound and SetupABO, we can build a DLIN distinguisher $\mathcal{B}$ with comparable running time such that $\mathbf{A d v}_{\mathcal{D}}^{\text {Setup-indist }}(\lambda) \leq 4 \cdot \mathbf{A d v}_{\mathcal{B}}^{\text {DIN }}(\lambda)$.

To prove the latter inequality, we first prove the indistinguishability of the two possible distributions for $\overrightarrow{g_{3}}$ (the case of $\overrightarrow{f_{3}}$ can be handled in a completely analogous way). To this end, we define an intermediate setup procedure Setuplnt which produces vectors of the form $\left(\overrightarrow{g_{1}}, \overrightarrow{g_{2}}, \overrightarrow{g_{3}}\right)$ with $\overrightarrow{g_{1}}=\left(g_{1}, 1, g\right), \overrightarrow{g_{2}}=\left(1, g_{2}, g\right), \overrightarrow{g_{3}}=\left(g_{3,1}, g_{3,2}, g_{3,3}\right)$ where $g_{1}, g_{2}, g_{3,1}, g_{3,2}, g_{3,3} \stackrel{R}{\leftarrow} \mathbb{G}$. The indistinguishability of the two distributions of $\left(\overrightarrow{g_{1}}, \overrightarrow{g_{2}}, \overrightarrow{g_{3}}\right)$ is obtained by combining the following two claims, the proofs of which are straightforward but given for completeness.

Claim 2. If DLIN holds, no PPT adversary can distinguish the output of SetupSound from Setuplnt.

Proof. We show a distinguisher $\mathcal{B}$ that takes in $\left(g, g_{1}, g_{2}, g_{1}^{\delta_{1}}, g_{2}^{\delta_{2}}, \chi\right)$, for some $\delta_{1}, \delta_{2} \stackrel{R}{\leftarrow} \mathbb{Z}_{p}$, with the purpose of deciding if $\chi=g^{\delta_{1}+\delta_{2}}$ or $\chi \in_{R} \mathbb{G}$. To this end, $\mathcal{B}$ defines $\overrightarrow{g_{1}}=\left(g_{1}, 1, g\right), \overrightarrow{g_{2}}=\left(1, g_{2}, g\right)$. As for $\overrightarrow{g_{3}}, \mathcal{B}$ defines it as $\overrightarrow{g_{3}}=\left(g_{1}^{\delta_{1}}, g_{2}^{\delta_{2}}, \chi\right)$. It is clear that, if $\chi=g^{\delta_{1}+\delta_{2}},\left(\overrightarrow{g_{1}}, \overrightarrow{g_{2}}, \overrightarrow{g_{3}}\right)$ is distributed as an output of SetupSound whereas, if $\chi \in_{R} \mathbb{G}$, it is an output of Setuplnt. 
Claim 3. If DLIN holds, no PPT adversary can distinguish the outputs of Setuplnt and SetupABO.

Proof. Consider a distinguisher $\mathcal{B}$ that takes as input $\left(g, g_{1}, g_{2}, g_{1}^{\delta_{1}}, g_{2}^{\delta_{2}}, \chi\right)$ and decides if $\chi=g^{\delta_{1}+\delta_{2}}$ or $\chi \in \in_{R} \mathbb{G}$. To do so, $\mathcal{B}$ defines $\overrightarrow{g_{1}}=\left(g_{1}, 1, g\right)$ and $\overrightarrow{g_{2}}=\left(1, g_{2}, g\right)$. As for the third vector $\overrightarrow{g_{3}}, \mathcal{B}$ and computes $\overrightarrow{g_{3}}=\left(g_{1}^{\delta_{1}}, g_{2}^{\delta_{2}}, \chi \cdot g^{-\operatorname{tag}^{\star}}\right)$. If $\chi \in_{R} \mathbb{G}$, the vector $\overrightarrow{g_{3}}$ has the same distribution no matter if $\chi$ is multiplied by $g^{-\operatorname{tag}^{\star}}$ or not and its distribution corresponds to that of an output of Setuplnt. If $\chi=g^{\delta_{1}+\delta_{2}}, \overrightarrow{g_{3}}$ is distributed as in parameters produced by SetupABO.

By repeating the same arguments for the two distributions of $\left(\vec{f}_{1}, \vec{f}_{2}, \vec{f}_{3}\right)$, we obtain the announced upper bound $\operatorname{Adv}_{\mathcal{D}}^{\text {Setup-indist }}(\lambda) \leq 4 \cdot \mathbf{A d v}_{\mathcal{B}}^{\text {DLIN }}(\lambda)$.

Correctness and Public Evaluability on $\mathcal{V}$. This property is implied by the public evaluability of the underlying standard hash proof system. Namely, for any element $\Phi \in \mathcal{V}$, which is a triple of the form $\left(\Phi_{1}, \Phi_{2}, \Phi_{3}\right)=\left(g_{1}^{\theta_{1}}, g_{2}^{\theta_{2}}, g^{\theta_{1}+\theta_{2}}\right)$ and for each $i$, the value $K_{i}=\Phi_{1}^{P_{1}(i)} \cdot \Phi_{2}^{P_{2}(i)} \cdot \Phi_{3}^{P(i)}$ equals $Y_{i, 1}^{\theta_{1}} \cdot Y_{i, 2}^{\theta_{2}}$ and is uniquely defined by pk and $\mathrm{vk}_{i}=\left(Y_{i, 1}, Y_{i, 2}\right)$. It is also immediate that combining any $t$ values $K_{i}=\Phi_{1}^{P_{1}(i)} \cdot \Phi_{2}^{P_{2}(i)} \cdot \Phi_{3}^{P(i)}$ allows recovering $X_{1}^{\theta_{1}} X_{2}^{\theta_{2}}$.

UnIVERSALITY. Let $\Phi=\left(\Phi_{1}, \Phi_{2}, \Phi_{3}\right)$ be a random triple in $\mathbb{G}^{3}$. With overwhelming probability, we have $\Phi_{3} \neq g^{\theta_{1}+\theta_{2}}$, where $\theta_{1}=\log _{g_{1}}\left(\Phi_{1}\right)$ and $\theta_{2}=\log _{g_{2}}\left(\Phi_{2}\right)$. For any $(t-1)$-subset $\bar{S} \subset\{1, \ldots, n\}$, given shares $\left\{\text { sk }_{i}=\left(P_{1}(i), P_{2}(i), P(i)\right)\right\}_{i \in \bar{S}}$ and public elements $X_{1}=g_{1}^{x_{1}} \cdot g^{z}, X_{2}=g_{2}^{x_{2}} \cdot g^{z}$, $\left\{\mathrm{vk}_{i}=\left(Y_{i, 1}, Y_{i, 2}\right)=\left(g_{1}^{P_{1}(i)} g^{P(i)}, g_{2}^{P_{2}(i)} g^{P(i)}\right)\right\}_{i=1}^{n}$, the value $z=P(0)$ is completely undetermined. Since $\Phi$ can be written $\left(\Phi_{1}, \Phi_{2}, \Phi_{3}\right)=\left(g_{1}^{\theta_{1}}, g_{2}^{\theta_{2}}, g^{\theta_{1}+\theta_{2}+\theta}\right)$ for some non-zero $\theta \in_{R} \mathbb{Z}_{p}^{*}$, its private evaluation can be expressed as

$$
\operatorname{PrivEval}\left(\mathrm{pk},\left\{\mathrm{sk}_{i}\right\}_{i=1}^{t}, \Phi\right)=\Phi_{1}^{x_{1}} \cdot \Phi_{2}^{x_{2}} \cdot \Phi_{3}^{z}=X_{1}^{\theta_{1}} \cdot X_{2}^{\theta_{2}} \cdot g^{\theta \cdot z}
$$

which is uniformly random since $z$ is itself random and independent of publicly available elements.

All-But-One Soundness. Algorithm SetupABO chooses $\left(\overrightarrow{g_{1}}, \overrightarrow{g_{2}}, \overrightarrow{g_{3}}\right)$ in such a way that, for any $\operatorname{tag} \neq \operatorname{tag}^{\star}$, the vector $\vec{g}_{\text {tag }}=\overrightarrow{g_{3}} \cdot(1,1, g)^{\operatorname{tag}}={\overrightarrow{g_{1}}}^{\xi_{1}} \cdot{\overrightarrow{g_{2}}}^{\xi_{2}} \cdot(1,1, g)^{\operatorname{tag}-\operatorname{tag}^{\star}}$ is not in $\operatorname{span}\left(\overrightarrow{g_{1}}, \overrightarrow{g_{2}}\right)$ and $\left(\overrightarrow{g_{1}}, \overrightarrow{g_{2}}, \vec{g}_{\text {tag }}\right)$ forms a Groth-Sahai CRS for the perfect soundness setting. Consequently, for any tag $\operatorname{tag} \neq \operatorname{tag}^{\star}$, even an unbounded adversary would be unable to produce a convincing proof $\pi_{\text {LIN }}$ for an element $\Phi \notin \mathcal{V}$. At the same time, $\vec{g}_{\mathrm{tag}^{\star}}=\overrightarrow{g_{1}} \xi_{1} \cdot{\overrightarrow{g_{2}}}^{\xi_{2}}$ is such that $\left(\overrightarrow{g_{1}}, \overrightarrow{g_{2}}, \vec{g}_{\text {tag }}\right)$ is a Groth-Sahai CRS for the perfect WI setting, and the trapdoor $\left(\xi_{1}, \xi_{2}\right)$ makes it possible to generate simulated proofs $\pi_{\text {LIN }}$ for elements $\Phi=\left(\Phi_{1}, \Phi_{2}, \Phi_{3}\right) \in \mathbb{G}^{3}$ that can be outside the language $\mathcal{V}$ of linear tuples. Whenever $\left(g, g_{1}, g_{2}, \Phi_{1}, \Phi_{2}, \Phi_{3}\right)$ is actually a linear tuple, simulated proofs (see Appendix B.1 for details on how to construct them) are distributed exactly as the proofs that would be produced using real witnesses.

Simulatability of Share Proofs. In the public parameters produced by SetupABO, the vectors $\mathbf{f}=\left(\overrightarrow{f_{1}}, \overrightarrow{f_{2}}, \overrightarrow{f_{3}}\right)$ are chosen in such a way that $\vec{f}_{3}=\vec{f}_{1}{ }^{\phi_{1}} \cdot \vec{f}_{2}{ }^{\phi_{2}}$. This means that $\left(\phi_{1}, \phi_{2}\right)$ can be used as a trapdoor to generate simulated NIZK proofs $\pi_{K_{i}}=\left(\vec{C}_{P_{1}}, \vec{C}_{P_{2}}, \vec{C}_{P}, \pi_{K_{i}}^{\prime}\right)$ that committed exponents $\left(P_{1}(i), P_{2}(i), P(i)\right)$ satisfy the multi-exponentiation equations

$$
Y_{i, 1}=g_{1}^{P_{1}(i)} \cdot g^{P(i)}, \quad Y_{i, 2}=g_{2}^{P_{2}(i)} g^{P(i)}, \quad K_{i}=\Phi_{1}^{P_{1}(i)} \cdot \Phi_{2}^{P_{2}(i)} \cdot \Phi_{3}^{P(i)} .
$$

Namely, $\vec{C}_{P_{1}}, \vec{C}_{P_{2}}, \vec{C}_{P}$ are generated as commitments to 0 and the proof for (5) is simulated using $\left(\phi_{1}, \phi_{2}\right)$. The resulting proof $\pi_{K_{i}}^{\prime}$ - which is a simulated proof for a true statement - has the same 
distribution as a real proof.

Consistency. This property holds unconditionally. This is implied by the perfect soundness of Groth-Sahai proofs. Namely, SetupSound produces common reference strings $\mathbf{g}_{\text {tag }}=\left(\overrightarrow{g_{1}}, \overrightarrow{g_{2}}, \overrightarrow{g_{\text {tag }}}\right)$ and $\mathbf{f}=\left(\vec{f}_{1}, \vec{f}_{2}, \vec{f}_{3}\right)$ that are always perfectly sound. This guarantees the impossibility of producing a convincing proof $\pi_{\mathrm{LIN}}$ for an element $\Phi=\left(\Phi_{1}, \Phi_{2}, \Phi_{3}\right)$ such that $\left(g, g_{1}, g_{2}, \Phi_{1}, \Phi_{2}, \Phi_{3}\right)$ is not a linear tuple. Moreover, thanks to the perfect soundness of proofs $\pi_{K_{i}}$ for the CRS $\mathbf{f}=\left(\vec{f}_{1}, \vec{f}_{2}, \vec{f}_{3}\right)$, invalid private evaluation shares $K_{i}$ can never be accepted by the ShareEvalVerify algorithm. Consequently, there is no way for two distinct sets of acceptable private evaluation shares to yield two distinct private evaluations for a valid $\Phi \in \mathcal{V}$.

Subset Membership Hardness. We show that no PPT adversary can distinguish the distributions $D_{0}:=\left\{\left(\mathrm{pk},\left\{\left(\mathrm{sk}_{i}, \mathrm{vk}_{i}\right)\right\}_{i=1}^{n}, \mathcal{C}, \mathcal{V}, C, \tau\right) \mid C \stackrel{R}{\leftarrow} \mathcal{V}\right\}$ and

$$
D_{1}:=\left\{\left(\mathrm{pk},\left\{\left(\mathrm{sk}_{i}, \mathrm{vk}_{i}\right)\right\}_{i=1}^{n}, \mathcal{C}, \mathcal{V}, C, \tau\right) \mid C \stackrel{R}{\leftarrow} \mathcal{C} \backslash \mathcal{V}\right\}
$$

so long as the DLIN assumption holds. Namely, any subset membership adversary $\mathcal{A}$ implies a DLIN distinguisher $\mathcal{B}^{\text {DLIN }}$ such that $\operatorname{Adv}_{\mathcal{A}}^{\mathrm{SM}}(\lambda) \leq \mathbf{A d v}_{\mathcal{B}^{\mathrm{DLIN}}}^{\mathrm{DLIN}}(\lambda)$.

The reduction $\mathcal{B}^{\text {DLIN }}$ takes as input a DLIN instance

$$
\left(g, g_{1}, g_{2}, \Phi_{1}=g_{1}^{\theta_{1}}, \Phi_{2}=g_{2}^{\theta_{2}}, \Phi_{3}=g^{\theta_{1}+\theta_{2}+\theta_{3}}\right),
$$

where $\theta_{1}, \theta_{2} \stackrel{R}{-} \mathbb{Z}_{p}$, with the task of deciding if $\theta_{3}=0$ or $\eta_{3} \in_{R} \mathbb{Z}_{p}$. It chooses random polynomials $P_{1}[Z], P_{2}[Z], P[Z]$ of degree $t-1$ which will be use to share $P_{1}(0)=x_{1}, P_{2}(0)=x_{2}$ and $P(0)=z$. Using these, it defines

$$
\mathrm{vk}_{i}=\left(Y_{i, 1}, Y_{i, 2}\right)=\left(g_{1}^{P_{1}(i)} g^{P(i)}, g_{2}^{P_{2}(i)} g^{P(i)}\right), \quad \mathbf{s k}_{i}=\left(P_{1}(i), P_{2}(i), P(i)\right) \quad \forall i \in\{1, \ldots, n\}
$$

and

$$
\mathrm{pk}=\left(\left(\mathbb{G}, \mathbb{G}_{T}\right), g, \overrightarrow{g_{1}}, \overrightarrow{g_{2}}, \overrightarrow{g_{3}}, \overrightarrow{f_{1}}, \overrightarrow{f_{2}}, \overrightarrow{f_{3}}, X_{1}=g_{1}^{x_{1}} \cdot g^{z}, X_{2}=g_{2}^{x_{2}} \cdot g^{z}\right),
$$

where $\vec{g}_{3}=\vec{g}_{1}^{\xi_{1}} \cdot \vec{g}_{2}^{\xi_{2}} \cdot(1,1, g)^{- \text {tag }^{\star}}$ and $\vec{f}_{3}=\vec{f}_{1}^{\phi_{1}} \cdot \vec{f}_{2}^{\phi_{2}}$ are chosen as in step 2 of SetupABO. The distribution of pk and $\left\{\left(\mathrm{sk}_{i}, \mathrm{vk}_{i}\right)\right\}_{i=1}^{n}$ is thus identical to the output distribution of $\operatorname{SetupABO}\left(\lambda, t, n, \operatorname{tag}^{\star}\right)$. As for the challenge value $C \in \mathcal{C}, \mathcal{B}^{\text {DLIN }}$ defines $C=\left(\Phi_{1}, \Phi_{2}, \Phi_{3}\right), \tau=\left(\xi_{1}, \xi_{2}, \phi_{1}, \phi_{2}\right)$ and outputs $D:=\left(\right.$ pk, $\left.\left\{\left(\mathrm{sk}_{i}, \mathrm{vk}_{i}\right)\right\}_{i=1}^{n}, \mathcal{C}, \mathcal{V}, C, \tau\right)$. By construction, if $\theta_{3}=0, D$ is a random sample from the distribution $D_{0}$. If $\theta_{3} \in R \mathbb{Z}_{p}$, we have $\theta_{3} \neq 0$ (i.e., $C \in \mathcal{C} \backslash \mathcal{V}$ ) with overwhelming probability, so that $D$ corresponds to the distribution $D_{1}$.

As mentioned earlier, our schemes only require secure erasures on behalf of the trusted dealer. Namely, the servers are not required to reliably erase their inputs. In order to prove the security of our DLIN-based instantiation without assuming erasures, the servers should be able to explain the generation of their non-interactive proofs $\pi_{K_{i}}$ for the statement (5) when the adversary chooses to corrupt them. In the proof of Theorem 1, the corrupted servers can faithfully reveal their internal state in all games, except for $\mathrm{Game}_{6}$ and $\mathrm{Game}_{7}$. In these games, the proofs $\pi_{K_{i}}$ of correct partial decryption are simulated without using the actual witnesses $\left(P_{1}(i), P_{2}(i), P(i)\right)$. However, since the CRS $\mathbf{f}=\left(\vec{f}_{1}, \vec{f}_{2}, \vec{f}_{3}\right)$ is perfectly hiding, the simulator has the flexibility to trapdoor open the fake commitments $\left(\vec{C}_{P_{1}}, \vec{C}_{P_{2}}, \vec{C}_{P}\right)$ to any value - including the real witnesses $\left(P_{1}(i), P_{2}(i), P(i)\right)$ - and provide random coins that plausibly explain $\pi_{K_{i}}$.

Consequently, our DLIN-based construction only requires a suitable distributed key generation protocol to completely eliminate the need for erasures. 


\subsection{An Instantiation from the SXDH Assumption in Prime Order Groups}

The construction of Section 5.2 relies on a well-established assumption in prime order groups and it is described in terms of symmetric pairings for simplicity. However, it readily extends to asymmetric pairing configurations.

Further efficiency improvements can be obtained if we choose to rely on asymmetric pairings $e: \mathbb{G} \times \hat{\mathbb{G}} \rightarrow \mathbb{G}_{T}$ and the Symmetric eXternal Diffie-Hellman assumption (SXDH), which posits that the DDH problem is hard in $\mathbb{G}$ and $\hat{\mathbb{G}}$ when $\mathbb{G} \neq \hat{\mathbb{G}}$ and no isomorphism is efficiently computable between $\mathbb{G}$ and $\hat{\mathbb{G}}$.

In this case, the public key comprises group elements $\left(g_{1}, g_{2}, X\right) \in \mathbb{G}$ with $X=g_{1}^{x_{1}} g_{2}^{x_{2}}$ and where $\left(x_{1}, x_{2}\right) \stackrel{R}{\leftarrow}\left(\mathbb{Z}_{p}\right)^{2}$ is part of the private key. The public key also includes vectors $\left(\vec{u}_{1}, \vec{u}_{2}\right)$, where $\vec{u}_{1}=(\hat{g}, \hat{h}) \in \hat{\mathbb{G}}^{2}$ and $\vec{u}_{2}=\vec{u}_{1}^{\rho_{u}}=\left(\hat{g}^{\rho_{u}}, \hat{h}^{\rho_{u}}\right) \in \hat{\mathbb{G}}^{2}$, for some $\rho_{u} \stackrel{R}{-} \mathbb{Z}_{p}$. It finally contains vectors $\left(\vec{v}_{1}, \vec{v}_{2}\right)$, where $\vec{v}_{1}=\left(v_{1,1}, v_{1,2}\right) \in \hat{\mathbb{G}}^{2}$ and $\vec{v}_{2}=\vec{v}_{1}^{\rho_{v}} \cdot(1, \hat{g})$, for some $\rho_{v} \stackrel{R}{\leftarrow} \mathbb{Z}_{p}$. These vectors $\left(\vec{v}_{1}, \vec{v}_{2}\right)$ are the counterpart of $\left(\vec{f}_{1}, \vec{f}_{2}, \vec{f}_{3}\right)$ in Section 5.2 and they form the CRS that allows generating proofs of well-formedness for private evaluation shares.

$\operatorname{SetupSound}(\lambda, t, n)$ : Choose a configuration of asymmetric bilinear groups $\left(\mathbb{G}, \hat{\mathbb{G}}, \mathbb{G}_{T}\right)$ of prime order $p>2^{\lambda}$ with generators $g_{1}, g_{2} \stackrel{R}{\leftarrow} \mathbb{G}$ and $\hat{g}, \hat{h} \stackrel{R}{\leftarrow} \hat{\mathbb{G}}$.

1. Choose $x_{1}, x_{2} \stackrel{R}{\leftarrow} \mathbb{Z}_{p}$ and set $X=g_{1}^{x_{1}} g_{2}^{x_{2}}$. Define vectors $\left(\vec{u}_{1}, \vec{u}_{2}\right)$, where $\vec{u}_{1}=(\hat{g}, \hat{h}) \in \hat{\mathbb{G}}^{2}$ and $\vec{u}_{2}=\vec{u}_{1}^{\rho_{u}}=\left(\hat{g}^{\rho_{u}}, \hat{h}^{\rho_{u}}\right) \in \hat{\mathbb{G}}^{2}$, for some $\rho_{u} \stackrel{R}{\leftarrow} \mathbb{Z}_{p}$.

2. Choose $v_{1,1}, v_{1,2} \stackrel{R}{\leftarrow} \hat{\mathbb{G}}$ and define the vectors $\left(\vec{v}_{1}, \vec{v}_{2}\right)$, where $\vec{v}_{1}=\left(v_{1,1}, v_{1,2}\right) \in \hat{\mathbb{G}}^{2}$ and $\vec{v}_{2}=\vec{v}_{1}^{\rho_{v}} \cdot(1, \hat{g})$, for some $\rho_{v} \stackrel{R}{\leftarrow} \mathbb{Z}_{p}$.

3. Choose random polynomials $P_{1}[Z], P_{2}[Z] \in \mathbb{Z}_{p}[Z]$ of degree $t-1$ such that $P_{1}(0)=x_{1}$ and $P_{2}(0)=x_{2}$. For each $i \in\{1, \ldots, n\}$, compute $Y_{i}=g_{1}^{P_{1}(i)} g_{2}^{P_{2}(i)}$.

4. Define private key shares $\mathbf{S K}=\left(\mathbf{s k}_{1}, \ldots, \mathbf{s k}_{n}\right)$ as $\mathbf{s k}_{i}=\left(P_{1}(i), P_{2}(i)\right) \in\left(\mathbb{Z}_{p}\right)^{2}$ for each $i \in\{1, \ldots, n\}$. Verification keys $\mathbf{V K}=\left(\mathrm{vk}_{1}, \ldots, \mathrm{vk}_{n}\right)$ are set as $\mathbf{v k}_{i}=Y_{i} \in \mathbb{G}$ for each $i$ and the public key is defined as

$$
\mathrm{pk}=\left(\left(\mathbb{G}, \hat{\mathbb{G}}, \mathbb{G}_{T}\right), g, \vec{u}_{1}, \vec{u}_{2}, \vec{v}_{1}, \vec{v}_{2}, X\right) .
$$

The sets $\left(\mathcal{C}, \mathcal{K}, \mathcal{K}^{\prime}, \mathcal{R}\right)$, they are defined as $\mathcal{C}=\mathbb{G}^{2}, \mathcal{K}=\mathcal{K}^{\prime}=\mathbb{G}$ and $\mathcal{R}=\mathbb{Z}_{p}$, respectively. The subset $\mathcal{V} \subset \mathcal{C}$ consists of the language $\left(\Phi_{1}, \Phi_{2}\right) \in \mathbb{G}^{2}$ for which there exists $\theta \in \mathbb{Z}_{p}$ such that $\Phi_{1}=g_{1}^{\theta}$ and $\Phi_{2}=g_{2}^{\theta}$.

$\operatorname{SetupABO}\left(\lambda, t, n, \operatorname{tag}^{\star}\right)$ : is identical to SetupSound with the following differences.

1. In step $1, \overrightarrow{u_{2}}$ is set as $\vec{u}_{2}=\vec{u}_{1}^{\rho_{u}} \cdot(1, \hat{g})^{-\operatorname{tag}^{\star}}$ instead of being chosen in $\operatorname{span}\left(\overrightarrow{g_{1}}, \overrightarrow{g_{2}}\right)$.

2 . In step 2 , the vectors $\left(\vec{v}_{1}, \vec{v}_{2}\right)$ are chosen so as to have $\vec{v}_{2}=\vec{v}_{1}^{\rho_{v}}$.

3. The algorithm additionally outputs the trapdoor $\tau=\left(\rho_{u}, \rho_{v}\right) \in\left(\mathbb{Z}_{p}\right)^{2}$.

Sample(pk): choose $\theta \stackrel{R}{\leftarrow} \mathbb{Z}_{p}$ and compute a pair $\Phi=\left(\Phi_{1}, \Phi_{2}\right)=\left(g_{1}^{\theta}, g_{2}^{\theta}\right)$. Then, output $(\theta, \Phi)$.

Prove $\left(\right.$ pk, tag, $\left.\left(\theta_{1}, \theta_{2}\right), \Phi\right)$ : parse pk as $\left(\left(\mathbb{G}, \hat{\mathbb{G}}, \mathbb{G}_{T}\right), g, \vec{u}_{1}, \vec{u}_{2}, \vec{v}_{1}, \vec{v}_{2}, X\right)$ and $\Phi$ as $\left(\Phi_{1}, \Phi_{2}\right) \in \mathbb{G}^{2}$. Construct a vector $\vec{u}_{\text {tag }}=\vec{u}_{2} \cdot(1, \hat{g})^{\text {tag }}$ and use $\mathbf{u}_{\text {tag }}=\left(\vec{u}_{1}, \vec{u}_{\text {tag }}\right)$ as a Groth-Sahai CRS to generate a NIZK proof that $\left(g, g_{1}, g_{2}, \Phi_{1}, \Phi_{2}\right)$ is a Diffie-Hellman tuple. More precisely, generate a commitment $\vec{C}_{\theta}$ to $\theta \in \mathbb{Z}_{p}$ (in other words, compute $\vec{C}_{\theta}=\vec{u}_{\text {tag }}^{\theta} \cdot{\overrightarrow{u_{1}}}^{r}$ with $r \stackrel{R}{\leftarrow} \mathbb{Z}_{p}$ for each $i \in\{1,2\}$ ) and a proof $\pi_{\mathrm{DH}}$ that it satisfies

$$
\Phi_{1}=g_{1}^{\theta}, \quad \Phi_{2}=g_{2}^{\theta} .
$$


The entire proof $\pi_{\mathrm{DH}}$ for (6) consists of $\vec{C}_{\theta}$ and $\pi_{\theta}$ (see Appendix B.2 for details about the generation of this proof) and requires 2 elements of $\widehat{\mathbb{G}}$ and 2 elements of $\mathbb{G}$.

SimProve $(\mathrm{pk}, \tau, \operatorname{tag}, \Phi)$ : parses pk as above, $\tau$ as $\left(\rho_{u}, \rho_{v}\right) \in\left(\mathbb{Z}_{p}\right)^{2}$ and $\Phi$ as $\left(\Phi_{1}, \Phi_{2}\right) \in \mathbb{G}^{2}$. If $\operatorname{tag} \neq \operatorname{tag}^{\star}$, return $\perp$. Otherwise, the commitment $\vec{C}_{\theta}$ and the proof $\pi_{\mathrm{DH}}$ must be generated for the CRS $\mathbf{u}_{\mathrm{tag}^{\star}}=\left(\vec{u}_{1}, \vec{u}_{\mathrm{tag}^{\star}}\right)$, where $\vec{u}_{\mathrm{tag}^{\star}}=\vec{u}_{2} \cdot(1, \hat{g})^{\mathrm{tag}}{ }^{\star}=\vec{u}_{1}^{\rho_{u}}$, which is a Groth-Sahai CRS for the perfect WI setting. The algorithm thus proceeds as follows.

1. Using the trapdoor $\rho_{u}$, simulate proofs for multi-exponentiation equations. That is, generate $\vec{C}_{\theta}$ as a commitment to 0 and compute $\pi_{\theta}$ as a simulated proof for relations (6).

2. Output $\pi_{\mathrm{DH}}=\left(\vec{C}_{\theta}, \pi_{\theta}\right)$ that consists of perfectly hiding commitments and simulated NIZK proofs which, on the CRS $\mathbf{u}_{\mathrm{tag}}{ }^{\star}=\left(\vec{u}_{1}, \vec{u}_{\mathrm{tag}}\right)$, have the same distribution as real proofs.

Verify $\left(\mathrm{pk}, \operatorname{tag}, \Phi, \pi_{\mathrm{DH}}\right)$ : parse pk and $\Phi$ as above and $\pi_{\mathrm{DH}}$ as $\left(\vec{C}_{\theta}, \pi_{\theta}\right) \in \hat{\mathbb{G}}^{2} \times \mathbb{G}^{2}$. Then, compute the vector $\vec{u}_{\text {tag }}=\vec{u}_{2} \cdot(1, \hat{g})^{\text {tag }}$ and use $\mathbf{u}_{\text {tag }}=\left(\vec{u}_{1}, \vec{u}_{\text {tag }}\right)$ as a Groth-Sahai CRS to verify the proof $\pi_{\mathrm{DH}}$. If the latter is deemed as a valid proof for relations (6), return 1. Otherwise, return 0.

PubEval $\left(\mathrm{pk},\left(\theta_{1}, \theta_{2}\right), \Phi\right)$ : parse pk and $\Phi$ as above. Return $\perp$ if $\left(\Phi_{1}, \Phi_{2}\right) \neq\left(g_{1}^{\theta}, g_{2}^{\theta}\right)$. Otherwise, compute and return $K=X^{\theta} \in \mathcal{K}$.

SharePrivEval $\left(\mathrm{pk}, \mathrm{sk}_{i}, \Phi\right)$ : parse $\mathrm{sk}_{i}$ as $\left(P_{1}(i), P_{2}(i)\right) \in\left(\mathbb{Z}_{p}\right)^{2}$ and return $\perp$ if $\Phi \notin \mathbb{G}^{2}$. Otherwise, return $\left(K_{i}, \pi_{K_{i}}\right)$, where $K_{i}=\Phi_{1}^{P_{1}(i)} \cdot \Phi_{2}^{P_{2}(i)} \in \mathcal{K}^{\prime}$ and $\pi_{K_{i}}=\left(\vec{C}_{P_{1}}, \vec{C}_{P_{2}}, \pi_{K_{i}}^{\prime}\right) \in \hat{\mathbb{G}}^{4} \times \mathbb{G}^{4}$ is a proof consisting of commitments $\vec{C}_{P_{1}}, \vec{C}_{P_{2}}$ to exponents $P_{1}(i), P_{2}(i) \in \mathbb{Z}_{p}$ and a proof $\pi_{K_{i}}^{\prime}$ that they satisfy the equations

$$
K_{i}=\Phi_{1}^{P_{1}(i)} \cdot \Phi_{2}^{P_{2}(i)}, \quad Y_{i}=g_{1}^{P_{1}(i)} \cdot g_{2}^{P_{2}(i)}
$$

The perfectly binding commitments $\vec{C}_{P_{1}}, \vec{C}_{P_{2}}$ and the proof $\pi_{K_{i}}^{\prime}$ are generated using the vectors $\mathbf{v}=\left(\vec{v}_{1}, \vec{v}_{2}\right)$ as a Groth-Sahai CRS .

ShareEvalVerify $\left(\mathrm{pk}, \mathrm{vk}_{i}, \Phi, K_{i}, \pi_{K_{i}}\right):$ parse $\mathrm{vk}_{i}$ as $Y_{i} \in \mathbb{G}$ and return $\perp$ in the event that $\left(K_{i}, \pi_{K_{i}}\right)$ cannot be parsed as a sequence of elements in $\mathbb{G} \times \hat{\mathbb{G}}^{4} \times \mathbb{G}^{4}$. Otherwise, parse the proof $\pi_{K_{i}}$ as $\pi_{K_{i}}=\left(\vec{C}_{P_{1}}, \vec{C}_{P_{2}}, \pi_{K_{i}}^{\prime}\right) \in \hat{\mathbb{G}}^{4} \times \mathbb{G}^{4}$ and return 1 if $\pi_{K_{i}}^{\prime}$ is a valid proof for equations (7). In any other situation, return 0 .

Combine $\left(\mathrm{pk}, \Phi,\left\{\left(K_{i}, \pi_{K_{i}}\right)\right\}_{i \in S}\right)$ : return $\perp$ if ShareEvalVerify $\left(\mathrm{pk}, \mathrm{vk}_{i}, \Phi, K_{i}, \pi_{K_{i}}\right)=0$ for some $i \in S$. Otherwise, compute $K=\prod_{i \in S} K_{i}^{\Delta_{i, S}(0)}=\Phi_{1}^{x_{1}} \cdot \Phi_{2}^{x_{2}} \in \mathcal{K}$.

The proof of the following theorem is completely similar to the proof of Theorem 3 and omitted.

Theorem 4. The above construction is an all-but-one perfectly sound hash proof system assuming that the SXDH assumption holds in $(\mathbb{G}, \hat{\mathbb{G}})$.

When the generic construction of Section 4 is instantiated with the above all-but-one hash proof system, the resulting cryptosystem (which is described in Section 6 for completeness) can be seen as a combination between Damgård's ElGamal encryption scheme [28] (as it is described in [51]) with a non-interactive one-time simulation-sound proof of validity of the ciphertext. The latter allows publicly verifying the validity of ciphertexts so as to achieve security in the threshold setting.

As detailed in Appendix B.2, the proof $\pi_{\mathrm{DH}}$ consists of 2 elements of $\mathbb{G}$ and the commitment $\vec{C}_{\theta}$ requires 2 elements of $\hat{\mathbb{G}}$. 


\section{A Concrete Adaptively Secure CCA2-Secure Threshold Cryptosystem}

For clarity, this section describes the specific construction obtained by instantiating our framework of Section 4 with the SXDH-based all-but-one threshold hash proof system of Section 5.3.

$\operatorname{Keygen}(\lambda, t, n)$ :

1. Choose a configuration of asymmetric bilinear groups $\left(\mathbb{G}, \hat{\mathbb{G}}, \mathbb{G}_{T}\right)$ of prime order $p>2^{\lambda}$ with generators $g_{1}, g_{2} \stackrel{R}{\leftarrow} \mathbb{G}$ and $\hat{g}, \hat{h} \stackrel{R}{\leftarrow} \hat{\mathbb{G}}$.

2. Choose $x_{1}, x_{2} \stackrel{R}{\leftarrow} \mathbb{Z}_{p}$ and set $X=g_{1}^{x_{1}} g_{2}^{x_{2}}$. Define vectors $\left(\vec{u}_{1}, \vec{u}_{2}\right)$, where $\vec{u}_{1}=(\hat{g}, \hat{h}) \in \hat{\mathbb{G}}^{2}$ and $\vec{u}_{2}=\vec{u}_{1}^{\rho_{u}}=\left(\hat{g}^{\rho_{u}}, \hat{h}^{\rho_{u}}\right) \in \hat{\mathbb{G}}^{2}$, for some $\rho_{u} \stackrel{R}{\leftarrow} \mathbb{Z}_{p}$.

3. Choose $v_{1,1}, v_{1,2} \stackrel{R}{\leftarrow} \hat{\mathbb{G}}$ and define the vectors $\left(\vec{v}_{1}, \vec{v}_{2}\right)$, where $\vec{v}_{1}=\left(v_{1,1}, v_{1,2}\right) \in \hat{\mathbb{G}}^{2}$ and $\vec{v}_{2}=\vec{v}_{1}^{\rho_{v}} \cdot(1, \hat{g})$, for some $\rho_{v} \stackrel{R}{\leftarrow} \mathbb{Z}_{p}$.

4. Choose random polynomials $P_{1}[Z], P_{2}[Z] \in \mathbb{Z}_{p}[Z]$ of degree $t-1$ such that $P_{1}(0)=x_{1}$ and $P_{2}(0)=x_{2}$. For each $i \in\{1, \ldots, n\}$, compute $Y_{i}=g_{1}^{P_{1}(i)} g_{2}^{P_{2}(i)}$.

5. Choose a strongly unforgeable one-time signature $\Sigma=(\mathrm{Gen}, \mathrm{Sig}, \mathrm{Ver})$ of which all algorithms use public parameters pp containing the description of $\mathbb{G}$.

6. Define private key shares $\mathbf{S K}=\left(S K_{1}, \ldots, S K_{n}\right)$ as $S K_{i}=\left(P_{1}(i), P_{2}(i)\right) \in\left(\mathbb{Z}_{p}\right)^{2}$ for each $i \in\{1, \ldots, n\}$. Verification keys $\mathbf{V K}=\left(V K_{1}, \ldots, V K_{n}\right)$ are set as $V K_{i}=Y_{i} \in \mathbb{G}$ for each $i$ and the public key is defined as

$$
P K=\left(\left(\mathbb{G}, \hat{\mathbb{G}}, \mathbb{G}_{T}\right), g, \vec{u}_{1}, \vec{u}_{2}, \vec{v}_{1}, \vec{v}_{2}, X, \Sigma\right) .
$$

$\operatorname{Encrypt}(M, P K)$ : to encrypt a message $M \in \mathbb{G}$, conduct the following steps.

1. Generate a one-time signature key pair $(\mathrm{SSK}, \mathrm{SVK}) \leftarrow \Sigma$.Gen $(\lambda, \mathrm{pp})$.

2. Choose $\theta \stackrel{R}{\leftarrow} \mathbb{Z}_{p}$ and compute

$$
C_{0}=M \cdot X^{\theta}, \quad C_{1}=g_{1}^{\theta}, \quad C_{2}=g_{2}^{\theta} .
$$

3. Construct a vector $\vec{u}_{\mathrm{SVK}}=\vec{u}_{2} \cdot(1, \hat{g})^{\mathrm{SVK}}$ and use $\mathbf{u}_{\mathrm{SVK}}=\left(\vec{u}_{1}, \vec{u}_{\mathrm{SVK}}\right)$ as a Groth-Sahai CRS to generate a NIZK proof that $\left(g_{1}, g_{2}, C_{1}, C_{2}\right)$ form a Diffie-Hellman tuple. More precisely, generate a commitment $\vec{C}_{\theta}$ to the encryption exponent $\theta \in \mathbb{Z}_{p}$ (in other words, compute $\vec{C}_{\theta}=\vec{u}_{\mathrm{SVK}}^{\theta} \cdot \vec{u}_{1}^{r}$ with $r \stackrel{R}{\leftarrow} \mathbb{Z}_{p}$ and a proof $\pi_{\mathrm{DH}}$ that they satisfy

$$
C_{1}=g_{1}^{\theta}, \quad C_{2}=g_{2}^{\theta} .
$$

The whole proof for (8) consists of $\vec{C}_{\theta}$ and $\pi_{\mathrm{DH}}$ (see Appendix B.2 for details about the generation of this proof).

4. Output the ciphertext

$$
C=\left(\mathrm{SVK}, C_{0}, C_{1}, C_{2}, \vec{C}_{\theta}, \pi_{\mathrm{DH}}, \sigma\right),
$$

where $\sigma=\Sigma \cdot \operatorname{Sig}\left(\mathrm{SSK},\left(C_{0}, C_{1}, C_{2}, \vec{C}_{\theta}, \pi_{\mathrm{DH}}\right)\right)$.

Ciphertext-Verify $(P K, C)$ : parse the ciphertext $C$ as (SVK, $\left.C_{0}, C_{1}, C_{2}, \vec{C}_{\theta}, \pi_{\mathrm{DH}}, \sigma\right)$. Return 1 if $\Sigma . \operatorname{Ver}\left(\mathrm{SVK},\left(C_{0}, C_{1}, C_{2}, \vec{C}_{\theta}, \pi_{\mathrm{DH}}\right), \sigma\right)=1$ and if $\pi_{\mathrm{DH}}$ is a valid proof for equations (8) and the Groth-Sahai CRS $\mathbf{u}_{\mathrm{SVK}}=\left(\vec{u}_{1}, \vec{u}_{2} \cdot(1, \hat{g})^{\mathrm{SVK}}\right)$. Otherwise, return 0 . 
Share-Decrypt $\left(S K_{i}, C\right)$ : on inputs $S K_{i}=\left(P_{1}(i), P_{2}(i)\right) \in \mathbb{Z}_{p}^{2}$ and $C$, return $(i, \perp)$ in the event that Ciphertext-Verify $(P K, C)=0$. Otherwise, compute $\hat{\mu}_{i}=\left(\nu_{i}, \vec{C}_{P_{1}}, \vec{C}_{P_{2}}, \pi_{\mu_{i}}\right)$ which consists of a partial decryption $\nu_{i}=C_{1}^{P_{1}(i)} \cdot C_{2}^{P_{2}(i)}$, commitments $\vec{C}_{P_{1}}, \vec{C}_{P_{2}}$ to $\left(P_{1}(i), P_{2}(i)\right) \in \mathbb{Z}_{p}^{2}$ and a proof $\pi_{\nu_{i}}$ that these satisfy the equations

$$
\nu_{i}=C_{1}^{P_{1}(i)} \cdot C_{2}^{P_{2}(i)}, \quad Y_{i, 1}=g_{1}^{P_{1}(i)} g_{2}^{P_{2}(i)}
$$

The commitments $\vec{C}_{P_{1}}, \vec{C}_{P_{2}}$ and the proof $\pi_{\nu_{i}}$ are generated using the CRS $\mathbf{v}=\left(\vec{v}_{1}, \vec{v}_{2}\right)$, so that $\vec{C}_{P_{j}}=\vec{v}_{2}^{P_{j}(i)} \cdot \vec{v}_{1}^{r_{j}}$ is a perfectly binding commitment, for some $r_{P_{j}} \stackrel{R}{-} \mathbb{Z}_{p}$, for each $j \in\{1,2\}$.

Share-Verify $\left(P K, C,\left(i, \hat{\mu}_{i}\right)\right)$ : parse $C$ as (SVK, $\left.C_{0}, C_{1}, C_{2}, \vec{C}_{\theta}, \pi_{\mathrm{DH}}, \sigma\right)$. If $\hat{\mu}_{i}=\perp$ or $\hat{\mu}_{i}$ cannot be properly parsed as $\left(\nu_{i}, \vec{C}_{P_{1}}, \vec{C}_{P_{2}}, \pi_{\mu_{i}}\right)$, return 0 . Otherwise, return 1 if $\pi_{\nu_{i}}$ is a valid proof. In any other situation, return 0 .

Combine $\left(P K, C,\left\{\left(i, \hat{\mu}_{i}\right)\right\}_{i \in S}\right)$ : for each $i \in S$, parse the share $\hat{\mu}_{i}$ as $\left(\nu_{i}, \vec{C}_{P_{1}}, \vec{C}_{P_{2}}, \pi_{\mu_{i}}\right)$ and return $\perp$ if Share-Verify $\left(P K, C,\left(i, \hat{\mu}_{i}\right)\right)=0$. Otherwise, compute $\nu=\prod_{i \in S} \nu_{i}^{\Delta_{i, S}(0)}$, which equals

$$
\nu=C_{1}^{x_{1}} \cdot C_{2}^{x_{2}}=X^{\theta}
$$

and in turn reveals $M=C_{0} / \nu$.

The ciphertext overhead now amounts to the length of 9 elements of $\mathbb{G}$ and 2 elements of $\hat{\mathbb{G}}$ if the one-time signature $\Sigma$ is instantiated using Groth's one-time signature [38], which is recalled in Appendix A.2. Using a family of curves with embedding degree 16 suggested by Kachisa et al. [48], we can assume that each element of $\mathbb{G}$ (resp. $\hat{\mathbb{G}}$ ) has a 340-bit (resp. 1020-bit) representation at the 128-bit security level when the results of [53] are taken into account. In this case, the ciphertext overhead reduces to 5100 bits.

From a computational point of view, if we assume that a multi-exponentiation with two base elements has roughly the same cost as a single exponentiation, the sender has to compute 8 exponentiations in $\mathbb{G}$ and 2 exponentiations in $\hat{\mathbb{G}}$. The validity of a ciphertext can be verified using only 6 pairing evaluations via a probabilistic batch-verification process as in [11].

An interesting application of this construction is its possible use in thresholdizing the opening procedure in group signatures (where group members verifiably encrypt their credentials under the public key of an opening authority) using Groth-Sahai proofs (e.g., [39]) while proving anonymity in a model where distributed opening authorities can be adaptively corrupted.

From a security point of view, we obtain the following result as a corollay of Theorem 1.

Theorem 5. The above threshold cryptosystem is IND-CCA secure against adaptive corruptions assuming that: (i) The SXDH assumption holds in $\left(\mathbb{G}, \hat{\mathbb{G}}, \mathbb{G}_{T}\right)$; (ii) $\Sigma$ is a strongly unforgeable one-time signature. Namely, for any adversary $\mathcal{A}$ making $Q$ decryption queries within running time $t$, there exist $D D H$ adversaries $\mathcal{D}_{1}\left(\right.$ resp. $\left.\mathcal{D}_{2}\right)$ in $\mathbb{G}$ (resp. $\hat{\mathbb{G}}$ ) and a one-time signature forger $\mathcal{D}_{3}$, which run in time $t^{\prime} \approx t+Q \cdot \operatorname{poly}(\lambda)$ and such that

$$
\mathbf{A d v}(\mathcal{A}) \leq \operatorname{Adv}_{\mathcal{D}_{1}}^{\mathrm{DDH}}(\lambda)+4 \cdot \mathbf{A d v}_{\mathcal{D}_{2}}^{\mathrm{DDH}}(\lambda)+\mathbf{A d v}_{\mathcal{D}_{3}}^{\mathrm{OTS}}(\lambda)+\frac{1}{2^{\lambda}}
$$




\section{Conclusion}

This paper described a framework for the construction of adaptively secure, robust and optimally resilient non-interactive CCA-secure threshold cryptosystems with tight security proofs in the standard model in the single-challenge setting. We showed instantiations based on simple assumptions in bilinear groups of composite and prime order.

The core of the framework consists of a suitable method for publicly verifying the validity of ciphertexts in encryption schemes built on hash proof systems. To solve this problem, we suggested a new notion of tag-based proof systems that yields very efficient one-time simulation-sound noninteractive proofs. These are of independent interest as their realizations in prime order groups readily extend to provide more efficient instantiations of the Naor-Yung encryption paradigm in the standard model.

It would be interesting to see if the new framework can be instantiated outside bilinear groups without appealing to random oracles. For example, it would be nice to have instantiations based on computational (instead of decisional) assumptions. Another interesting open problem is to define a similar framework for adaptively secure threshold signatures. While Wee's framework [73] covers the signature case, it focuses on the static corruption setting.

\section{References}

1. M. Abdalla, F. Ben Hamouda, D. Pointcheval. Disjunctions for Hash Proof Systems: New Constructions and Applications. In Eurocrypt'15, LNCS 9057, pp. 69-100, Springer, 2015.

2. M. Abe. Robust Distributed Multiplicaton with out Interaction. In Crypto'99, LNCS 1666, pp. 130-147, 1999.

3. M. Abe, B. David, M. Kohlweiss, R. Nishimaki, and M. Ohkubo. Tagged one-time signatures: Tight security and optimal tag size. K. Kurosawa and G. Hanaoka, editors, PKC 2013. Springer, 2013.

4. M. Abe, Y. Cui, H. Imai, E. Kiltz. Efficient Hybrid Encryption from ID-Based Encryption. In Designs Codes and Cryptography, 54(3), pp. 205-240, 2010.

5. M. Abe, S. Fehr. Adaptively Secure Feldman VSS and Applications to Universally-Composable Threshold Cryptography. In Crypto'04, LNCS 3152, pp. 317-334, 2004.

6. M. Abe, G. Fuchsbauer, J. Groth, K. Haralambiev, and M. Ohkubo. Structure-preserving signatures and commitments to group elements. T. Rabin, editor, Crypto 2010. Springer, 2010.

7. J. Almansa, I. Damgård, J.-B. Nielsen. Simplified Threshold RSA with Adaptive and Proactive Security. In Eurocrypt'06, LNCS 4004, pp. 593-611, 2006.

8. P. Barreto, M. Naehrig. Pairing-Friendly Elliptic Curves of Prime Order. In $S A C^{\prime} 05$, LNCS 3897, pp. 319-331, 2005.

9. M. Bellare, A. Boldyreva, S. Micali. Public-key encryption in a multi-user setting: Security proofs and improvements. In Eurocrypt '00, LNCS 1807, pp. 259-274, Springer, 2000.

10. M. Bellare, P. Rogaway. Random oracles are practical: A paradigm for designing efficient protocols. In $A C M$ CCS, pp. 62-73, 1993.

11. O. Blazy, G. Fuchsbauer, M. Izabachène, A. Jambert, H. Sibert, D. Vergnaud. Batch Groth-Sahai. In Applied Cryptography and Network Security (ACNS'10), LNCS 6123, pp. 218-235, 2010.

12. D. Boneh, X. Boyen. Efficient Selective-ID Secure Identity-Based Encryption Without Random Oracles. In Eurocrypt'04, LNCS 3027, pp. 223-238, 2004.

13. D. Boneh, X. Boyen, S. Halevi. Chosen Ciphertext Secure Public Key Threshold Encryption Without Random Oracles. In CT-RSA'06, LNCS 3860, pp. 226-243, 2006.

14. D. Boneh, X. Boyen, H. Shacham. Short group signatures. In Crypto'04, LNCS 3152, pp. 41-55, 2004.

15. D. Boneh, M. Franklin. Identity-Based Encryption from the Weil Pairing. In SIAM J. of Computing 32(3), pp. 586-615, 2003. Earlier version in Crypto'01.

16. D. Boneh, E.-J. Goh, K. Nissim. Evaluating 2-DNF formulas on ciphertexts. In Theory of Cryptography Conference - TCC 2005, LNCS 3378, pp. 325-341. Springer, 2005.

17. C. Boyd. Digital Multisignatures. In Cryptography and Coding (H.J. Beker and F.C. Piper Eds.), Oxford University Press, pp. 241-246, 1989. 
18. X. Boyen, Q. Mei, B. Waters. Direct Chosen Ciphertext Security from Identity-Based Techniques. in $A C M$ CCS'05, pp. 320-329, 2005.

19. J. Camenisch, N. Chandran, and V. Shoup. A public key encryption scheme secure against key dependent chosen plaintext and adaptive chosen ciphertext attacks. In Eurocrypt'09, LNCS 5479, pages 351-368, 2009.

20. J. Camenisch, K. Haralambiev, M. Kohlweiss, J. Lapon, V. Naessens. Structure Preserving CCA Secure Encryption and Applications. In Asiacrypt 2011, LNCS 7073, pp. 89-106, 2011

21. R. Canetti, R. Gennaro, S. Jarecki, H. Krawczyk, T. Rabin. Adaptive Security for Threshold Cryptosystems. In Crypto'99, LNCS 1666, pp. 98-115, 1999.

22. R. Canetti, S. Goldwasser. An Efficient Threshold Public Key Cryptosystem Secure Against Adaptive Chosen Ciphertext Attack. In Eurocrypt'99, LNCS 1592, pp. 90-106, 1999.

23. R. Canetti, S. Halevi, J. Katz. Chosen-Ciphertext Security from Identity-Based Encryption. In Eurocrypt'04, LNCS 3027, pp. 207-222, 2004.

24. R. Cramer, I. Damgård, S. Dziembowski, M. Hirt, T. Rabin. Efficient Multi-Party Computations Secure Against an Adaptive Adversary. In Eurocrypt'99, LNCS 1592, pp. 311-326, 1999.

25. R. Cramer, I. Damgård, Y. Ishai. Share Conversion, Pseudorandom Secret-Sharing and Applications to Secure Computation. In TCC'05, LNCS 3378, pp. 342-362, 2005.

26. R. Cramer, V. Shoup. A practical public key cryptosystem provably secure against adaptive chosen ciphertext attack. In Crypto'98, LNCS 1462, pp. 13-25, 1998.

27. R. Cramer, V. Shoup. Universal Hash Proofs and a Paradigm for Adaptive Chosen Ciphertext Secure Public-Key Encryption. In Eurocrypt'02, LNCS 2332, pp. 45-64, 2002.

28. I. Damgård. Towards Practical Public Key Systems Secure Against Chosen Ciphertext Attacks. In Crypto'91, LNCS 576, pp. 445-456 1991.

29. Y. Dodis, N. Fazio. Public Key Trace and Revoke Scheme Secure against Adaptive Chosen Ciphertext Attack. In PKC'03, LNCS 293, LNCS 2567, pp. 100-115, 2003.

30. Y. Desmedt. Society and Group Oriented Cryptography: A New Concept. In Crypto'87, LNCS 293, pp. 120-127, 1987.

31. Y. Desmedt, Y. Frankel. Threshold Cryptosystems. In Crypto'89, LNCS 435, pp. 307-315, 1989.

32. Y. Dodis, J. Katz. Chosen-Ciphertext Security of Multiple Encryption. In TCC'05, LNCS 3378, pp. 188-209, 2005.

33. P.-A. Fouque, D. Pointcheval. Threshold Cryptosystems Secure against Chosen-Ciphertext Attacks. In Asiacrypt'01, LNCS 2248, pp. 351-368, 2001.

34. Y. Frankel, P. MacKenzie, M. Yung. Adaptively-Secure Distributed Public-Key Systems. In ESA'99, LNCS 1643, pp. 4-27, 1999.

35. Y. Frankel, P. MacKenzie, M. Yung. Adaptively-Secure Optimal-Resilience Proactive RSA. In Asiacrypt'99, LNCS 1716, pp. 180-194, 1999.

36. D. Freeman. Converting Pairing-Based Cryptosystems from Composite-Order Groups to Prime-Order Groups. In Eurocrypt'10, LNCS 6110, pp. 44-61, 2010.

37. J. Groth, R. Ostrovsky, A. Sahai. Perfect non-interactive zero knowledge for NP. In Eurocrypt'06, volume 4004 of Lecture Notes in Computer Science, pages 339-358. Springer, 2006.

38. J. Groth. Simulation-sound NIZK proofs for a practical language and constant size group signatures. In Asiacrypt 2006, LNCS 4284, pp. 444-459, 2006.

39. J. Groth. Fully anonymous group signatures without random oracles. In Asiacrypt 2007, LNCS 4833, pp. 164-180. Springer, 2007.

40. J. Groth, A. Sahai. Efficient non-interactive proof systems for bilinear groups. In Eurocrypt'08, LNCS 4965, pp. 415-432, 2008.

41. J. Håstad, R. Impagliazzo, L. Levin, M. Luby. A pseudorandom generator from any one-way function. SIAM Journal on Computing, vol. 28(4), pp. 1364-1396, 1999.

42. D. Hofheinz, T. Jager. Tightly secure signatures and public-key encryption. In Crypto '12, LNCS 7417, pp. 590607, Springer, 2012.

43. D. Hofheinz, E. Kiltz. The Group of Signed Quadratic Residues and Applications. In Crypto'09, LNCS 5677, pp. 637-653, 2009.

44. S. Jarecki, A. Lysyanskaya. Adaptively Secure Threshold Cryptography: Introducing Concurrency, Removing Erasures. In Eurocrypt'00, LNCS 1807, pp. 221-242, 2000.

45. C. Jutla, A. Roy. Relatively-Sound NIZKs and Password-Based Key-Exchange. In PKC 2012, LNCS 7293, pp. 485-503, 2012.

46. C. Jutla, A. Roy. Shorter Quasi-Adaptive NIZK Proofs for Linear Subspaces. In Asiacrypt 2013, LNCS 8269, pp. 1-20, 2013. 
47. C. Jutla, A. Roy. Switching lemma for bilinear tests and constant-size NIZK proofs for linear subspaces. In Crypto '14, LNCS 8617, pp. 295-312, Springer, 2014.

48. E. Kachisa, E. Schaefer, M. Scott. Constructing Brezing-Weng Pairing-Friendly Elliptic Curves Using Elements in the Cyclotomic Field. In Pairing 2008, LNCS 5209, pp. 126-135, 2008.

49. J. Katz, V. Vaikuntanathan. Round-Optimal Password-Based Authenticated Key Exchange. In TCC'11, LNCS 6597, pp. 293-310, 2011.

50. E. Kiltz. Chosen-ciphertext security from tag-based encryption. In TCC'06, LNCS 3876, pp. 581-600, 2006.

51. E. Kiltz, K. Pietrzak, M. Stam, M. Yung. A New Randomness Extraction Paradigm for Hybrid Encryption. In Eurocrypt'09, LNCS 5479, pp. 590-609, 2009.

52. E. Kiltz, H. Wee. Quasi-Adaptive NIZK for Linear Subspaces Revisited. In Eurocrypt 2015, LNCS 9057, pp. $101-128,2015$.

53. T. Kim, R. Barbulescu. Extended Tower Number Field Sieve: A New Complexity for the Medium Prime Case. In Crypto 2016, LNCS 9814, pp. 543-571, 2016.

54. A. Lewko, B. Waters. New Techniques for Dual System Encryption and Fully Secure HIBE with Short Ciphertexts. In TCC 2010, LNCS 5978, pp. 455-479, 2010.

55. B. Libert, T. Peters, M. Joye, M. Yung. Non-malleability from Malleability: Simulation-Sound Quasi-Adaptive NIZK Proofs and CCA2-Secure Encryption from Homomorphic Signatures. In Eurocrypt 2014, LNCS 8441, pp. 514-532, 2014.

56. B. Libert, T. Peters, C. Qian. Structure-Preserving Chosen-Ciphertext Security with Shorter Verifiable Ciphertexts. In PKC 2017, LNCS 10174, pp. 247-276, 2017.

57. B. Libert, M. Yung. Adaptively Secure Non-Interactive Threshold Cryptosystems. In ICALP 2011, LNCS 6756, pp. 588-600, 2011.

58. P. MacKenzie. An Efficient Two-Party Public Key Cryptosystem Secure against Adaptive Chosen Ciphertext Attack. In PKC'03, LNCS 2567, pp. 47-61, 2003.

59. P. MacKenzie, M. Reiter, K. Yang. Alternatives to non-malleability: Definitions, constructions, and applications. In TCC'04, LNCS 2951, pp. 171-190. Springer, 2004.

60. T. Malkin, I. Teranishi, Y. Vahlis, M. Yung. Signatures resilient to continual leakage on memory and computation. In TCC'11, LNCS 6597, pp. 89-106, 2011.

61. M. Naor, M. Yung. Public-key cryptosystems provably secure against chosen ciphertext attacks. In STOC'90, ACM Press, 1990.

62. R. Ostrovsky, M. Yung. How to Withstand Mobile Virus Attacks. In $10^{\text {th }}$ ACM Symp. on Principles of Distributed Computing (PODC'91), 1991.

63. T. Rabin. A Simplified Approach to Threshold and Proactive RSA. In Crypto'98, LNCS 1462, pp. 89-104, 1998.

64. C. Rackoff, D. Simon. Non-Interactive Zero-Knowledge Proof of Knowledge and Chosen Ciphertext Attack. In Crypto'91, LNCS 576, pp. 433-444, 1991.

65. A. Sahai. Non-Malleable Non-Interactive Zero Knowledge and Adaptive Chosen-Ciphertext Security. In FOCS'99, pp. 543-553, 1999.

66. M. Scott. Authenticated ID-based Key Exchange and remote log-in with simple token and PIN number. Cryptology ePrint Archive: Report 2002/164.

67. A. Shamir. Identity-Based Cryptosystems and Signature Schemes. In Crypto'84, LNCS 196, pp. 47-53, 1984.

68. V. Shoup, R. Gennaro. Securing Threshold Cryptosystems against Chosen Ciphertext Attack. In J. of Cryptology, 15(2), pp. 75-96, 2002. Earlier version in Eurocrypt'98, LNCS 1403, pp. 1-16, 1998.

69. Z. Wang, H. Qian, Z. Li. Adaptively Secure Threshold Signature Scheme in the Standard Model. In Informatica 20(4), pp. 591--612, 2009.

70. B. Waters. Efficient Identity-Based Encryption Without Random Oracles. In Eurocrypt'05, LNCS 3494, 2005.

71. B. Waters. Dual System Encryption: Realizing Fully Secure IBE and HIBE under Simple Assumptions. In Crypto'09, LNCS 5677, pp. 619-636, 2009.

72. H. Wee. Efficient Chosen-Ciphertext Security via Extractable Hash Proofs. In Crypto'10, LNCS 6223, pp. 314-332, 2010.

73. H. Wee. Threshold and Revocation Cryptosystems via Extractable Hash Proofs. In Eurocrypt'11, LNCS 6632, pp. 589-609, 2011.

\section{A One-time Signatures}

\section{A.1 Definition}

A one-time signature scheme is a triple of algorithms $\Sigma=$ (Gen, Sig, Ver) such that, on input of a security parameter $\lambda$ and (optionally) a set of externally supplied public parameters pp, $\mathcal{G}$ generates 
a one-time key pair $(\mathrm{SSK}, \mathrm{SVK}) \leftarrow \Sigma \cdot \mathcal{G}(\lambda, \mathrm{pp})$ while, for any message $M, \Sigma \cdot \operatorname{Ver}(\mathrm{SVK}, M, \sigma)$ outputs 1 whenever $\sigma=\Sigma$.Sig $(\mathrm{SSK}, M)$ and 0 otherwise.

As in [23,32], we need strongly unforgeable one-time signatures: no PPT adversary can be able to create a new signature for a previously signed message.

Definition 4. $\Sigma=(\mathrm{Gen}, \mathrm{Sig}, \mathrm{Ver})$ is a strongly unforgeable one-time signature if the probability

$$
\begin{aligned}
\mathbf{A d v}^{\mathrm{OTS}}=\operatorname{Pr}[ & (\mathrm{SSK}, \mathrm{SVK}) \leftarrow \mathcal{G}(\lambda, \mathrm{pp}) ;(M, S t) \leftarrow \mathcal{F}(\mathrm{SVK}) ; \\
& \sigma \leftarrow \Sigma \cdot \operatorname{Sig}(\mathrm{SSK}, M) ;\left(M^{\prime}, \sigma^{\prime}\right) \leftarrow \mathcal{F}(M, \sigma, \mathrm{SVK}, S t): \\
& \left.\Sigma \cdot \operatorname{Ver}\left(\sigma^{\prime}, \mathrm{SVK}, M^{\prime}\right)=1 \wedge\left(M^{\prime}, \sigma^{\prime}\right) \neq(M, \sigma)\right],
\end{aligned}
$$

where St denotes the state information maintained by $\mathcal{F}$ between stages, is negligible for any PPT forger $\mathcal{F}$.

\section{A.2 Groth's One-Time Signature}

For completeness, this section recalls the description of the one-time signature proposed by Groth [38], which was proved strongly unforgeable under the Discrete Logarithm assumption. The description assumes pre-existing public parameters pp consisting of a cyclic group $\mathbb{G}$ of prime order $p>2^{\lambda}$ with a generator $g \in \mathbb{G}$ and a random member $H:\{0,1\}^{*} \rightarrow \mathbb{Z}_{p}$ of collision-resistant hash function family.

$\operatorname{Gen}(\lambda, \mathrm{pp})$ : Given $\lambda \in \mathbb{N}$, a key pair is generated by taking the following steps.

1. Choose $x, y \stackrel{R}{\leftarrow} \mathbb{Z}_{p}$ and compute $f=g^{x}, h=g^{y}$.

2. Choose $r, s \stackrel{R}{-} \mathbb{Z}_{p}$ and compute $c=f^{r} h^{s}$.

Output SVK $=(f, h, c) \in \mathbb{G}^{3}$ and SSK $=(x, y, r, s) \in \mathbb{Z}_{p}^{4}$.

$\operatorname{Sig}(\mathrm{SSK}, M):$ To sign $M \in\{0,1\}^{*}$, choose $t \stackrel{R}{\leftarrow} \mathbb{Z}_{p}$ and compute

$$
\sigma=\left(t, \frac{x \cdot(r-t)+y \cdot s-H(M)}{y}\right) \in \mathbb{Z}_{p}^{2}
$$

$\operatorname{Ver}(\mathrm{SVK}, M, \sigma)$ : Given a message $M$ and a purported signature $\sigma=(t, w) \in \mathbb{Z}_{p}^{2}$, return 1 if

$$
c=g^{H(M)} \cdot f^{t} \cdot h^{w}
$$

and 0 otherwise.

\section{B Constructing Non-Interactive Proofs for Schemes in Prime Order Groups}

\section{B.1 Constructing Proof Elements in the DLIN-based Instantiation}

In the following notations, we define a coordinate-wise pairing $E: \mathbb{G} \times \mathbb{G}^{3} \rightarrow \mathbb{G}_{T}^{3}$ such that, for any element $h \in \mathbb{G}$ and any vector $\vec{g}=\left(g_{1}, g_{2}, g_{3}\right)$, we have $E(h, \vec{g})=\left(e\left(h, g_{1}\right), e\left(h, g_{2}\right), e\left(h, g_{3}\right)\right)$.

To construct the proof $\pi_{\text {LIN }}$ that $\Phi=\left(\Phi_{1}, \Phi_{2}, \Phi_{3}\right)=\left(g_{1}^{\theta_{1}}, g_{2}^{\theta_{2}}, g^{\theta_{1}+\theta_{2}}\right)$, for some $\left(\theta_{1}, \theta_{2}\right) \in\left(\mathbb{Z}_{p}\right)^{2}$, the sender first computes commitments $\vec{C}_{\theta_{i}}=\vec{g}_{\mathrm{tag}}^{\theta_{i}} \cdot{\overrightarrow{g_{1}}}^{r_{i}} \cdot{\overrightarrow{g_{2}}}^{s_{i}}=\left(g_{\mathrm{tag}, 1}^{\theta_{i}} \cdot g_{1}^{r_{i}}, g_{\mathrm{tag}, 2}^{\theta_{i}} \cdot g_{2}^{s_{i}}, g_{\mathrm{tag}, 3}^{\theta_{i}} \cdot g^{r_{i}+s_{i}}\right)$, 
for each $i \in\{1,2\}$, with $r_{1}, r_{2}, s_{1}, s_{2} \stackrel{R}{\leftarrow} \mathbb{Z}_{p}$ and where $\vec{g}_{\text {tag }}=\left(g_{\text {tag }, 1}, g_{\text {tag }, 2}, g_{\text {tag }, 3}\right) \in \mathbb{G}^{3}$. Then, he generates the proof $\pi_{\left(\theta_{1}, \theta_{2}\right)}$ as

$$
\pi_{\left(\theta_{1}, \theta_{2}\right)}=\left(\pi_{1}, \pi_{2}, \pi_{3}, \pi_{4}, \pi_{5}, \pi_{6}\right)=\left(g_{1}^{r_{1}}, g_{1}^{s_{1}}, g_{2}^{r_{2}}, g_{2}^{s_{2}}, g^{r_{1}+r_{2}}, g^{s_{1}+s_{2}}\right)
$$

which satisfies the verification equations

$$
\begin{aligned}
E\left(g_{1}, \vec{C}_{\theta_{1}}\right) & =E\left(\Phi_{1}, \vec{g}_{\mathrm{tag}}\right) \cdot E\left(\pi_{1}, \overrightarrow{g_{1}}\right) \cdot E\left(\pi_{2}, \overrightarrow{g_{2}}\right) \\
E\left(g_{2}, \vec{C}_{\theta_{2}}\right) & =E\left(\Phi_{2}, \vec{g}_{\mathrm{tag}}\right) \cdot E\left(\pi_{3}, \overrightarrow{g_{1}}\right) \cdot E\left(\pi_{4}, \overrightarrow{g_{2}}\right) \\
E\left(g, \vec{C}_{\theta_{1}} \cdot \vec{C}_{\theta_{2}}\right) & =E\left(\Phi_{3}, \vec{g}_{\mathrm{tag}}\right) \cdot E\left(\pi_{5}, \overrightarrow{g_{1}}\right) \cdot E\left(\pi_{6}, \overrightarrow{g_{2}}\right) .
\end{aligned}
$$

When the above verifications are performed in the naive way, they require to evaluate 30 pairings altogether. However, using randomized batch verification techniques (which, as illustrated in [11], can provide substantial savings in the context of Groth-Sahai proofs), they can be more efficiently processed by computing a product of 12 pairings at the expense of a tiny probability of accepting an invalid ciphertext.

On a CRS $\left(\overrightarrow{g_{1}}, \overrightarrow{g_{2}}, \vec{g}_{\text {tag }}\right)$ for the WI setting (i.e., where $\vec{g}_{\text {tag }}=\vec{g}_{1}{ }_{1} \cdot \vec{g}_{2} \xi_{2}$ for some $\xi_{1}, \xi_{2} \in R$ $\left.\mathbb{Z}_{p}\right)$, the proof $\pi_{\text {LIN }}$ can be simulated as follows. First, commitments $\vec{C}_{\theta_{1}}, \vec{C}_{\theta_{2}}$ are computed as commitments to 0 (say $\vec{C}_{\theta_{i}}={\overrightarrow{g_{1}}}^{r_{i}} \cdot{\overrightarrow{g_{2}}}^{s_{i}}$ for each $i \in\{1,2\}$ with $r_{1}, r_{2}, s_{1}, s_{2} \stackrel{R}{\leftarrow} \mathbb{Z}_{p}$ ). Then, proof elements $\pi_{\left(\theta_{1}, \theta_{2}\right)}=\left(\pi_{1}, \pi_{2}, \pi_{3}, \pi_{4}, \pi_{5}, \pi_{6}\right)$ satisfying (11) can be obtained as per

$$
\begin{array}{lll}
\pi_{1}=g_{1}^{r_{1}} \cdot \Phi_{1}^{-\xi_{1}} & \pi_{3}=g_{2}^{r_{2}} \cdot \Phi_{2}^{-\xi_{1}} & \pi_{5}=g^{r_{1}+r_{2}} \cdot \Phi_{3}^{-\xi_{1}} \\
\pi_{2}=g_{1}^{s_{1}} \cdot \Phi_{1}^{-\xi_{2}} & \pi_{4}=g_{2}^{s_{2}} \cdot \Phi_{2}^{-\xi_{2}} & \pi_{6}=g^{s_{1}+s_{2}} \cdot \Phi_{3}^{-\xi_{2}} .
\end{array}
$$

\section{B.2 Constructing Proof Elements in the SXDH-based instantiation}

Here, our notations use a coordinate-wise pairing $E: \mathbb{G} \times \hat{\mathbb{G}}^{2} \rightarrow \mathbb{G}_{T}^{2}$ such that, for any element $h \in \mathbb{G}$ and any vector $\vec{g}=\left(\hat{g_{1}}, \hat{g_{2}}\right) \in \hat{\mathbb{G}}^{2}$, we have $E(h, \vec{g})=\left(e\left(h, \hat{g_{1}}\right), e\left(h, \hat{g_{2}}\right)\right)$.

To construct the non-interactive proof $\pi_{\mathrm{DH}}$ that $\left(\Phi_{1}, \Phi_{2}\right)=\left(g_{1}^{\theta}, g_{2}^{\theta}\right)$, for some $\theta \in_{R} \mathbb{Z}_{p}$, the sender first computes a commitment $\vec{C}_{\theta}=\vec{u}_{\text {tag }}^{\theta} \cdot{\overrightarrow{u_{1}}}^{r}=\left(\hat{u}_{\text {tag }, 1}^{\theta} \cdot \hat{g}^{r}, \hat{u}_{\text {tag }, 2}^{\theta} \cdot \hat{h}^{r}\right)$, using a randomly drawn $r \stackrel{R}{\leftarrow} \mathbb{Z}_{p}$ and where $\vec{u}_{\text {tag }}=\left(\hat{u}_{\text {tag, }, 1}, \hat{u}_{\text {tag }, 2}\right) \in \hat{\mathbb{G}}^{2}$. Then, he generates the proof $\pi_{\theta}$ as

$$
\pi_{\theta}=\left(\pi_{1}, \pi_{2}\right)=\left(g_{1}^{r}, g_{2}^{r}\right) \in \mathbb{G}^{2}
$$

which satisfies the verification equations

$$
\begin{aligned}
& E\left(g_{1}, \vec{C}_{\theta}\right)=E\left(\Phi_{1}, \vec{u}_{\mathrm{tag}}\right) \cdot E\left(\pi_{1}, \overrightarrow{u_{1}}\right) \\
& E\left(g_{2}, \vec{C}_{\theta}\right)=E\left(\Phi_{2}, \vec{u}_{\mathrm{tag}}\right) \cdot E\left(\pi_{2}, \overrightarrow{u_{1}}\right) .
\end{aligned}
$$

Instead of naively verifying equations (12) separately, the verifier can choose $\omega \stackrel{R}{\leftarrow} \mathbb{Z}_{p}$ and test whether

$$
E\left(g_{1} \cdot g_{2}^{\omega}, \vec{C}_{\theta}\right)=E\left(\Phi_{1} \cdot \Phi_{2}^{\omega}, \vec{u}_{\mathrm{tag}}\right) \cdot E\left(\pi_{1} \cdot \pi_{2}^{\omega}, \vec{u}_{1}\right),
$$

which fails with overwhelming probability when one of the two equations (12) is not satisfied. With further optimizations (when coordinate-wise equalities are simultaneously batch-verified), the verifier only needs to compute a product of 6 pairings. 
On a CRS $\left(\vec{u}_{\text {tag }}, \vec{u}_{1}\right)$ for the perfect WI setting (i.e., where $\vec{u}_{\text {tag }}=\vec{u}_{1}^{\rho_{u}}$ for some $\rho_{u} \in \in_{R} \mathbb{Z}_{p}$ ), a NIZK proof $\pi_{\mathrm{DH}}$ can be simulated by computing $\vec{C}_{\theta}$ as a commitment to 0 (say $\vec{C}_{\theta}=\vec{u}_{1}^{r}$ for some $\left.r \stackrel{R}{\leftarrow} \mathbb{Z}_{p}\right)$ and the assignment

$$
\pi_{1}=g_{1}^{r} \cdot \Phi_{1}^{-\rho_{u}}=g_{2}^{r} \cdot \Phi_{2}^{-\rho_{u}}
$$

is easily seen to satisfy the verification equations (12). 